\title{
Towards frame-like gauge invariant formulation for massive mixed symmetry bosonic fields. II. General Young tableau with two rows
}

\author{
Yu. M. Zinoviev* \\ Institute for High Energy Physics \\ Protvino, Moscow Region, 142280, Russia
}

\begin{abstract}
In this paper we complete our construction of frame-like gauge invariant description for massive mixed symmetry tensor fields corresponding to arbitrary Young tableau with two rows started in [1]. We consider general massive theory in $(A) d S$ spaces with arbitrary cosmological constant as well as all special limits which exist both in de Sitter and in anti-de Sitter spaces.
\end{abstract}

\footnotetext{
*E-mail address: Yurii.Zinoviev@ihep.ru
} 


\section{Introduction}

In this paper we complete our construction of frame-like gauge invariant description for massive mixed symmetry tensor fields corresponding to arbitrary Young tableau with two rows started in [1]. Frame-like formalism [2, 3, 4] is a natural generalization of well-known frame formulation of gravity in terms of veilbein $e_{\mu}{ }^{a}$ and Lorentz connection $\omega_{\mu}{ }^{a b}$ and it turns out to be very convenient for the investigation of possible interacting theories for higher spin particles as well as of gauge symmetry algebras behind them.

There are two different frame-like formulations for massless mixed symmetry bosonic fields. For simplicity, let us restrict ourselves with mixed symmetry tensors corresponding to Young tableau with two rows. Let us denote $Y(k, l)$ a tensor $\Phi^{a_{1} \ldots a_{k}, b_{1} \ldots b_{l}}$ which is symmetric both on first $k$ as well as last $l$ indices, completely traceless on all indices and satisfies a constraint $\Phi^{\left(a_{1} \ldots a_{k}, b_{1}\right) b_{2} \ldots b_{l}}=0$, where round brackets mean symmetrization. In a metric-like formulation such field has two gauge transformations with gauge parameters corresponding to $Y(k, l-1)$ and $Y(k-1, l)$ (the only exception being the case $k=l$ with one gauge symmetry with parameter $Y(k, k-1)$ only). In the first approach [5, 6, 7, 8] for the description of $Y(k, l)$ tensor $(k \neq l)$ one use a one-form $e_{\mu}^{Y(k-1, l)}$ as a main physical field. In this, only one of two gauge symmetries is realized explicitly and such approach is very well adapted for the $\operatorname{Ad} S$ spaces. Another formulation [9] uses two-form $e_{\mu \nu}^{Y(k-1, l-1)}$ as a main physical field in this, both gauge symmetries are realized explicitly. Such formalism works in flat Minkowski space while deformation into $A d S$ space requires introduction of additional fields [10]. Technical reason is that it is impossible to deform into $(A) d S$ space keeping both gauge symmetries, while physical reason is that massless fields in $(A) d S$ space in general have more physical degrees of freedom than in flat Minkowski space. As a byproduct of our investigation in Sections 2 and 3 we obtain appropriate sets of such additional physical fields both for $A d S$ and $d S$ spaces.

For completeness we give in Section 1 all necessary information on massless fields that we will need in the following sections for constructing massive theories. Also we consider in this section a possibility to deform such massless theories into $(A) d S$ space. In most cases such deformation is impossible but the structure of mass-like terms and corresponding corrections for gauge transformations will be heavily used in what follows.

Then we turn to the construction of gauge invariant formulation for massive fields. Recall that there are two general approaches to gauge invariant description of massive fields. One of them uses powerful BRST approach [11, 12, 13, 14, 15, 16, 17, 18. Another one, which we will follow in this work, [19, 20, 21, 22, 23, 1, 24, 25, 26] is a generalization to higher spin fields of well-known mechanism of spontaneous gauge symmetry breaking. In this, one starts with appropriate set of massless fields with all their gauge symmetries and obtain gauge invariant description for massive field as a smooth deformation. One of the nice features of gauge invariant formulation for massive fields is that it allows us effectively use all known properties of massless fields serving as building blocks. As we have already seen in all cases considered previously and we will see again in this paper, gauge invariant description of massive fields always admits smooth deformation into $(A) d S$ space without introduction of any additional fields besides those that are necessary in flat Minkowski space so that restriction mentioned above will not be essential for us.

In Section 2 we consider special case of $Y(k+1, k+1)$ tensor fields corresponding to 
rectangular Young tableau and then in Section 3 we consider general case $Y(k+1, l+1)$ with $k \neq l$. As we will see in both cases gauge invariance completely fixes all parameters in the Lagrangian and gauge transformations leaving us with one free parameter having dimension of mass only. It is hardly possible to give meaningful definition of what is mass for mixed symmetry (spin)-tensor fields in $(A) d S$ spaces and we will not insist on any such definition. Instead, we will simply use this parameter to analyze all possible special limits that exist in (A) $d S$ spaces. In this, only fields having the same number of degrees of freedom as massless one in flat Minkowski space we will call massless ones, while all other special limits that appear in $(A) d S$ spaces will be called partially massless $[27,28,29,20,30$.

\section{Massless fields}

In this section we provide all necessary information on massless fields that we will need in the following sections for constructing of massive ones. In all cases we consider a possibility to deform massless theory into $(A) d S$ space. As is well known [10], in general such deformation is impossible without introduction of additional fields, but the structure of mass-like terms and corresponding corrections for gauge transformations will be heavily used in the following sections. Note that all Lagrangians in this and the following sections will be completely antisymmetric on world indices, thus working in $(A) d S$ spaces we will need so-called Lorentz covariant derivatives acting on local indices only. Our conventions on such covariant derivatives will be:

$$
\left[D_{\mu}, D_{\nu}\right] \xi^{a}=-\kappa\left(e_{\mu}{ }^{a} \xi_{\nu}-e_{\nu}{ }^{a} \xi_{\mu}\right), \quad \kappa=\frac{2 \Lambda}{(d-1)(d-2)}
$$

\subsection{Tensor $Y(k+1,0)$}

Besides mixed symmetry tensor fields we will need frame-like formulation for completely symmetric tensor fields [2, 3] (see also [23]). The main physical field is a one form $\Phi_{\mu}{ }^{a_{1} \ldots a_{k}}=$ $\Phi_{\mu}{ }^{\left(a_{k}\right)}$ (here and in what follows we will use the same condensed notations for tensor objects as in our previous works on the subject [23, 1, 26]), completely symmetric and traceless on local indices and auxiliary one form $\omega_{\mu}^{a,\left(a_{k}\right)}$, symmetric on last $k$ indices, completely traceless on all local indices and satisfying a constraint $\omega_{\mu}^{\left(a, a_{k}\right)}=0$. The Lagrangian and gauge transformations for massless field have the form:

$$
\begin{aligned}
(-1)^{k} \mathcal{L}_{0}= & -\left\{\begin{array}{l}
\mu \nu \\
a b
\end{array}\right\}\left[\omega_{\mu}^{e, a\left(a_{k-1}\right)} \omega_{\nu}^{e, b\left(a_{k-1}\right)}+\frac{1}{k} \omega_{\mu}^{a,\left(a_{k}\right)} \omega_{\nu}^{b,\left(a_{k}\right)}\right]+ \\
& +2\left\{\begin{array}{c}
\mu \nu \alpha \\
a b c
\end{array}\right\} \omega_{\mu}^{a, b\left(a_{k-1}\right)} \partial_{\nu} \Phi_{\alpha}^{c\left(a_{k-1}\right)}
\end{aligned}
$$

where $\left\{\begin{array}{c}\mu \nu \\ a b\end{array}\right\}=e^{\mu}{ }_{a} e^{\nu}{ }_{b}-e^{\mu}{ }_{b} e^{\nu}{ }_{a}$ and so on,

$$
\delta_{0} \Phi_{\mu}^{\left(a_{k}\right)}=\partial_{\mu} \zeta^{\left(a_{k}\right)}+\chi_{\mu}^{\left(a_{k}\right)}, \quad \delta_{0} \omega_{\mu}^{a,\left(a_{k}\right)}=\partial_{\mu} \chi^{a,\left(a_{k}\right)}
$$

where parameters $\zeta$ and $\chi$ have the same properties on local indices as $\Phi$ and $\omega$, correspondingly. 
Now let us consider deformation into $A d S$ space. If we replace all derivatives in the Lagrangian and gauge transformations by the covariant ones, the Lagrangian cease to be invariant:

$$
\delta_{0} \mathcal{L}_{0}=(-1)^{k} \frac{2(k+1)(d+k-3)}{k} \kappa\left[\omega_{\mu}^{\mu,\left(a_{k}\right)} \zeta^{\left(a_{k}\right)}-\chi^{\mu,\left(a_{k}\right)} \Phi_{\mu}{ }^{\left(a_{k}\right)}\right]
$$

To compensate for this non-invariance we introduce mass-like terms into the Lagrangian:

$$
(-1)^{k} \Delta \mathcal{L}_{0}=b_{k}\left\{\begin{array}{l}
\mu \nu \\
a b
\end{array}\right\} \Phi_{\mu}^{a\left(a_{k-1}\right)} \Phi_{\nu}^{b\left(a_{k-1}\right)}
$$

as well as corresponding corrections for gauge transformations:

$$
\begin{aligned}
\delta_{2} \omega_{\mu}{ }^{a,\left(a_{k}\right)}= & \frac{b_{k}}{(k+1)(d-2)}\left[k e_{\mu}{ }^{a} \zeta^{\left(a_{k}\right)}-e_{\mu}{ }^{\left(a_{1}\right.} \zeta^{\left.a_{k-1}\right) a}+\right. \\
& \left.+\frac{1}{(d+k-3)}\left(2 g^{\left(a_{1} a_{2}\right.} \zeta_{\mu}^{\left.a_{k-2}\right) a}-(k-1) g^{a\left(a_{1}\right.} \zeta_{\mu}^{\left.a_{k-1}\right)}\right)\right]
\end{aligned}
$$

where $b_{k}=(k+1)(d+k-3) \kappa$.

\subsection{Tensor $Y(k+1,1)$}

This tensor turns out to be special and requires separate consideration [9, 1]. Main physical field now - two form $\Psi_{\mu \nu}{ }^{\left(a_{k}\right)}$ completely symmetric and traceless on all local indices, while auxiliary field is a one form $\Omega_{\mu}{ }^{\left(a_{k}\right), b c}$ symmetric on first $k$ indices, antisymmetric on last two ones, traceless on all local indices and satisfying a constraint $\Omega_{\mu}{ }^{\left(a_{k}, b\right) c}=0$. Lagrangian and gauge transformations for massless field have the form:

$$
\begin{aligned}
(-1)^{k} \mathcal{L}_{0}= & \left\{\begin{array}{c}
\mu \nu \\
a b
\end{array}\right\}\left[\Omega_{\mu}{ }^{a\left(a_{k-1}\right), c d} \Omega_{\nu}{ }^{b\left(a_{k-1}\right), c d}+\frac{2}{k} \Omega_{\mu}{ }^{\left(a_{k}\right), a c} \Omega_{\nu}{ }^{\left(a_{k}\right), b c}\right]- \\
& -\left\{\begin{array}{c}
\mu \nu \alpha \beta \\
a b c d
\end{array}\right\} \Omega_{\mu}{ }^{a\left(a_{k-1}\right), b c} \partial_{\nu} \Psi_{\alpha \beta}{ }^{d\left(a_{k-1}\right)} \\
\delta_{0} \Psi_{\mu \nu}{ }^{\left(a_{k}\right)}= & \partial_{[\mu} \xi_{\nu]}{ }^{\left(a_{k}\right)}+\eta^{\left(a_{k}\right)}{ }_{\mu \nu}, \quad \delta_{0} \Omega_{\mu}{ }^{\left(a_{k}\right), b c}=\partial_{\mu} \eta^{\left(a_{k}\right), b c}
\end{aligned}
$$

where parameters $\xi$ and $\eta$ have the same properties on local indices as $\Phi$ and $\Omega$, correspondingly.

Now, if we replace all derivatives in the Lagrangian and gauge transformations by the covariant ones, we obtain non-invariance of the form:

$$
(-1)^{k} \delta_{0} \mathcal{L}_{0}=\frac{2(k+2)(d+k-4)}{k} \kappa\left\{\begin{array}{l}
\mu \nu \\
a b
\end{array}\right\}\left[\eta^{\left(a_{k}\right), a b} \Psi_{\mu \nu}{ }^{\left(a_{k}\right)}-\Omega_{\mu}{ }^{\left(a_{k}\right), a b} \xi_{\nu}{ }^{\left(a_{k}\right)}\right]
$$

but in this case it is impossible to restore broken invariance (without introduction of additional fields) mainly because there is no covariant mass-like term for such field.

\subsection{Tensor $Y(k+1, k+1)$}

This case is also special and deserves separate consideration. Main physical field now two form $R_{\mu \nu}{ }^{\left(a_{k}\right),\left(b_{k}\right)}$ symmetric on both groups of local indices, completely traceless on all local indices and satisfying constraints $R_{\mu \nu}{ }^{\left(a_{k}\right),\left(b_{k}\right)}=R_{\mu \nu}{ }^{\left(b_{k}\right),\left(a_{k}\right)}$ and $R_{\mu \nu}{ }^{\left(a_{k}, b_{1}\right)\left(b_{k-1}\right)}=0$, while 
auxiliary field is a two form $\Omega_{\mu \nu}\left(a_{k}\right),\left(b_{k}\right), c$. The Lagrangian and gauge transformations for massless field have the form:

$$
\begin{aligned}
& \mathcal{L}_{0}=-\frac{1}{2}\left\{\begin{array}{c}
\mu \nu \alpha \beta \\
a b c d
\end{array}\right\}\left[\Omega_{\mu \nu}{ }^{a\left(a_{k-1}\right), b\left(b_{k-1}\right), e} \Omega_{\alpha \beta} c\left(a_{k-1}\right), d\left(b_{k-1}\right), e+\right. \\
& +\frac{2}{k} \Omega_{\mu \nu}\left(a_{k}\right), a\left(b_{k-1}\right), b \Omega_{\alpha \beta}\left(a_{k}\right), c\left(b_{k-1}\right), d+ \\
& +\left\{\begin{array}{l}
\mu \nu \alpha \beta \gamma \\
a b c d e
\end{array}\right\} \Omega_{\mu \nu}{ }^{a\left(a_{k-1}\right), b\left(b_{k-1}\right), c} \partial_{\alpha} R_{\beta \gamma} d\left(a_{k-1}\right), e\left(b_{k-1}\right) \\
& \delta R_{\mu \nu}{ }^{\left(a_{k}\right),\left(b_{k}\right)}=\partial_{[\mu} \xi_{\nu]}{ }^{\left(a_{k}\right),\left(b_{k}\right)}+\eta_{[\mu}{ }^{\left(a_{k}\right),\left(b_{k}\right)}{ }_{\nu]}, \quad \delta \Omega_{\mu \nu}{ }^{\left(a_{k}\right),\left(b_{k}\right), c}=\partial_{[\mu} \eta_{\nu]}{ }^{\left(a_{k}\right),\left(b_{k}\right), c}
\end{aligned}
$$

One of the peculiar features of this tensor is that it admits deformation into $A d S$ space without introduction of any additional fields. Indeed, non-invariance of the Lagrangian that appears if we replace all derivatives by the covariant ones looks as follows:

$$
\delta_{0} \mathcal{L}_{0}=\frac{4(k+2)(d+k-5)}{k} \kappa\left\{\begin{array}{c}
\mu \nu \alpha \\
a b c
\end{array}\right\}\left[\Omega_{\mu \nu}{ }^{\left(a_{k}\right), a\left(b_{k-1}\right), b} \xi_{\alpha}{ }^{\left(a_{k}\right), c\left(b_{k-1}\right)}+\eta_{\mu}{ }^{\left(a_{k}\right), a\left(b_{k-1}\right), b} R_{\nu \alpha}{ }^{\left(a_{k}\right), c\left(b_{k-1}\right)}\right]
$$

But the invariance can be restored by adding to the Lagrangian mass-like term of the form:

$$
\mathcal{L}_{2}=a_{k, k}\left\{\begin{array}{c}
\mu \nu \alpha \beta \\
a b c d
\end{array}\right\} R_{\mu \nu}^{a\left(a_{k-1}\right), b\left(b_{l-1}\right)} R_{\alpha \beta}{ }^{c\left(a_{k-1}\right), d\left(b_{l-1}\right)}
$$

as well as appropriate corrections for gauge transformations:

$$
\begin{aligned}
\delta_{2} \Omega_{\mu \nu}\left(a_{k}\right),\left(b_{k}\right), c=-\frac{2 a_{k, k}}{(k+2)(d-4)}[ & k e_{[\mu}{ }^{c} \xi_{\nu]}{ }^{\left(a_{k}\right),\left(b_{k}\right)}-e_{[\mu}{ }^{\left(a_{1}\right.} \xi_{\nu]}{ }^{\left.a_{k-1}\right) c,\left(b_{k}\right)}-e_{[\mu}{ }^{\left(b_{1}\right.} \xi_{\nu]}{ }^{\left.\left(a_{k}\right), b_{k-1}\right) c}- \\
-\frac{1}{(d+k-5)} & {\left[\left(g^{\left(a_{1} a_{2}\right.} \xi_{[\mu, \nu]}{ }^{\left.a_{k-2}\right) c,\left(b_{k}\right)}+g^{\left(b_{1} b_{2}\right.} \xi_{[\mu}{ }^{\left.\left(a_{k}\right), b_{k-2}\right) c}{ }_{\nu]}\right)-\right.} \\
& -(k-1)\left(g^{c\left(a_{1}\right.} \xi_{[\mu, \nu]}{ }^{\left.a_{k-1}\right),\left(b_{k}\right)}+g^{c\left(b_{1}\right.} \xi_{[\mu}{ }^{\left.\left(a_{k}\right), b_{k-1}\right)}{ }_{\nu]}\right)+ \\
& \left.\left.+g^{\left(a _ { 1 } \left(b_{1}\right.\right.}\left(\xi_{[\mu, \nu]}^{\left.\left.a_{k-1}\right), b_{k-1}\right) c}+\xi_{[\mu}{ }^{\left.\left.a_{k-1}\right) c, b_{k-1}\right)}{ }_{\nu]}\right)\right]\right]
\end{aligned}
$$

provided $a_{k, k}=\frac{(k+2)}{2}(d+k-5) \kappa$.

\subsection{Tensor $Y(k+1, l+1)$}

Now we are ready to consider general case $-Y(k+1, l+1), k>l>0$. Main physical field here - two form $\Psi_{\mu \nu}{ }^{\left(a_{k}\right),\left(b_{l}\right)}$ symmetric on both groups of local indices, completely traceless on all local indices and satisfying a constraint $\Psi_{\mu \nu}\left(a_{k}, b_{1}\right)\left(b_{l-1}\right)=0$, while auxiliary field is a two form $\Omega_{\mu \nu}\left(a_{k}\right),\left(b_{l}\right), c$. The Lagrangian and gauge transformations for massless field have the form:

$$
\begin{aligned}
& (-1)^{k+l} \mathcal{L}_{0}=-\frac{1}{2}\left\{\begin{array}{l}
\mu \nu \alpha \beta \\
a b c d
\end{array}\right\}\left[\Omega_{\mu \nu}^{a\left(a_{k-1}\right), b\left(b_{l-1}\right), e} \Omega_{\alpha \beta} c\left(a_{k-1}\right), d\left(b_{l-1}\right), e+\right. \\
& +\frac{1}{k} \Omega_{\mu \nu}{ }^{\left(a_{k}\right), a\left(b_{l-1}\right), b} \Omega_{\alpha \beta}\left(a_{k}\right), c\left(b_{l-1}\right), d+ \\
& \left.+\frac{1}{l} \Omega_{\mu \nu}{ }^{a\left(a_{k-1}\right),\left(b_{l}\right), b} \Omega_{\alpha \beta} c\left(a_{k-1}\right),\left(b_{l}\right), d\right]+ \\
& +\left\{\begin{array}{l}
\mu \nu \alpha \beta \gamma \\
a b c d e
\end{array}\right\} \Omega_{\mu \nu} a\left(a_{k-1}\right), b\left(b_{l-1}\right), c \partial_{\alpha} \Psi_{\beta \gamma} d\left(a_{k-1}\right), e\left(b_{l-1}\right)
\end{aligned}
$$




$$
\delta \Psi_{\mu \nu}{ }^{\left(a_{k}\right),\left(b_{l}\right)}=\partial_{[\mu} \xi_{\nu]}{ }^{\left(a_{k}\right),\left(b_{l}\right)}+\eta_{[\mu}{ }^{\left(a_{k}\right),\left(b_{l}\right)}{ }_{\nu]}, \quad \delta \Omega_{\mu \nu}{ }^{\left(a_{k}\right),\left(b_{l}\right), c}=\partial_{[\mu} \eta_{\nu]}{ }^{\left(a_{k}\right),\left(b_{l}\right), c}
$$

Non-invariance of the Lagrangian that appears if we replace all derivatives by the covariant ones looks as follows:

$$
\begin{aligned}
& (-1)^{k+l} \delta_{0} \mathcal{L}_{0}=2 \kappa\left\{\begin{array}{l}
\mu \nu \alpha \\
a b c
\end{array}\right\}\left[\frac{[(k+2)(d+k-4)+(d+l-6)]}{k} \Omega_{\mu \nu}{ }^{\left(a_{k}\right), a\left(b_{l-1}\right), b} \xi_{\alpha}{ }^{\left(a_{k}\right), c\left(b_{l-1}\right)}+\right. \\
& +\frac{(l+1)(d+l-6)}{l} \Omega_{\mu \nu}^{a\left(a_{k-1}\right),\left(b_{l}\right), b} \xi_{\alpha}{ }^{c\left(a_{k-1}\right),\left(b_{l}\right)}+ \\
& +\frac{[(k+2)(d+k-4)+(d+l-6)]}{k} \eta_{\mu}^{\left(a_{k}\right), a\left(b_{l-1}\right), b} \Psi_{\nu \alpha}^{\left(a_{k}\right), c\left(b_{l-1}\right)}+ \\
& \left.+\frac{(l+1)(d+l-6)}{l} \eta_{\mu}^{a\left(a_{k-1}\right),\left(b_{l}\right), b} \Psi_{\nu \alpha}^{c\left(a_{k-1}\right),\left(b_{l}\right)}\right]
\end{aligned}
$$

We could try to restore broken gauge invariance by adding mass-like terms to the Lagrangian:

$$
(-1)^{k+l} \mathcal{L}_{2}=a_{k, l}\left\{\begin{array}{c}
\mu \nu \alpha \beta \\
a b c d
\end{array}\right\} \Psi_{\mu \nu}^{a\left(a_{k-1}\right), b\left(b_{l-1}\right)} \Psi_{\alpha \beta}{ }^{c\left(a_{k-1}\right), d\left(b_{l-1}\right)}
$$

as well as appropriate corrections for gauge transformations:

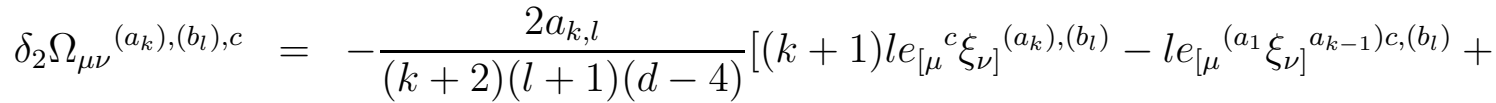

$$
\begin{aligned}
& \left.+e_{[\mu}{ }^{\left(a_{1}\right.} \xi_{\nu]}{ }^{\left.a_{k-1}\right)\left(b_{1}, b_{l-1}\right) c}-(k+1) e_{[\mu}{ }^{\left(b_{1}\right.} \xi_{\nu]}{ }^{\left.\left(a_{k}\right), b_{l-1}\right) c}+\ldots\right]
\end{aligned}
$$

where dots stand for the terms which are necessary to make transformations to be traceless (see Appendix), but this gives additional variations of the form:

$$
\begin{aligned}
&-4(-1)^{k+l} a_{k, l} \quad\left\{\begin{array}{c}
\mu \nu \alpha \\
a b c
\end{array}\right\} {\left[\frac{1}{k} \eta_{\mu}{ }^{\left(a_{k}\right), a\left(b_{l-1}\right), b} \Psi_{\nu \alpha}{ }^{\left(a_{k}\right), c\left(b_{l-1}\right)}+\frac{1}{l} \eta_{\mu}{ }^{a\left(a_{k-1}\right),\left(b_{l}\right), b} \Psi_{\nu \alpha}{ }^{c\left(a_{k-1}\right),\left(b_{l}\right)}+\right.} \\
&\left.+\frac{1}{k} \Omega_{\mu \nu}{ }^{\left(a_{k}\right), a\left(b_{l-1}\right), b} \xi_{\alpha}{ }^{\left(a_{k}\right), c\left(b_{l-1}\right)}+\frac{1}{l} \Omega_{\mu \nu}{ }^{a\left(a_{k-1}\right),\left(b_{l}\right), b} \xi_{\alpha}{ }^{c\left(a_{k-1}\right),\left(b_{l}\right)}\right]
\end{aligned}
$$

so that it is impossible to achieve a cancellation by adjusting the only free parameter $a_{k, l}$.

\section{Massive tensor $Y(k+1, k+1)$}

We have already seen in the previous section that tensor $Y(k+1, k+1)$ turns out to be special and deserves separate consideration. In this section we consider massive theory for such tensor. Recall that frame-like gauge invariant formulation for massive tensors $Y(k+1,0)$ and $Y(k+1,1)$ has been constructed in [23] and [1] correspondingly.

First of all, to construct gauge invariant formulation for massive field we have to determine a set of additional Goldstone fields required for such formulation. In general for each gauge symmetry of main gauge field we have to introduce corresponding primary Goldstone field. But these primary fields turn out to be gauge fields themselves with their own gauge symmetries so we have to introduce secondary fields and so on. In this, working with mixed symmetry (spin)-tensors one has to take into account reducibility of their gauge transformations. Let us illustrate the procedure on this particular case. Our main field $Y(k+1, k+1)$ 
has one gauge symmetry with the parameter corresponding to $Y(k+1, k)$ and this transformations are reducible with the reducibility parameter $Y(k, k)$. So we introduce primary field $Y(k+1, k)$. This field in turn has two gauge symmetries with parameters $Y(k+1, k-1)$ and $Y(k, k)$ and reducibility parameter $Y(k, k-1)$, thus taking into account reducibility of main field gauge transformations it is enough to introduce one secondary field $Y(k+1, k-1)$ only. It is not hard to see that the procedure stops at the field $Y(k+1,0)$ corresponding to completely symmetric tensor and having one gauge transformation with parameter $Y(k, 0)$ only. Thus the minimal set of fields necessary for gauge invariant description contains the fields $Y(k+1, n)$ with $(0 \leq n \leq k+1)$.

In frame-like gauge invariant formalism for massive bosonic fields the general structure of the Lagrangian looks as follows (schematically):

$$
\mathcal{L} \sim \mathcal{L}_{0}+\mathcal{L}_{1}+\mathcal{L}_{2}, \quad \mathcal{L}_{0} \sim \Omega \Omega+\Omega \partial \Phi, \quad \mathcal{L}_{1} \sim m \Omega \Phi, \quad \mathcal{L}_{2} \sim m^{2} \Phi \Phi
$$

where $\mathcal{L}_{0}$ and $\mathcal{L}_{2}$ are kinetic and mass terms for all fields involved, while $\mathcal{L}_{1}$ - a set of cross terms mixing different fields together (see below). Similarly, the general structure of gauge transformations looks like:

$\delta \sim \delta_{0}+\delta_{1}+\delta_{2}, \quad \delta_{0} \Phi \sim \partial \xi+\eta, \quad \delta_{0} \Omega \sim \partial \eta, \quad \delta_{1} \Phi \sim m \xi, \quad \delta_{1} \Omega \sim m \eta, \quad \delta_{2} \Omega \sim m^{2} \xi$

Thus it is convenient to organize the variations of the Lagrangian by the order of mass parameter $m$ (in a metric-like formulation this corresponds to the number of derivatives in variations). Taking into account that in flat Minkowski space our kinetic terms are already gauge invariant $\delta_{0} \mathcal{L}_{0}=0$, while in $(A) d S$ spaces they give non-trivial contributions of order $m^{2}$ (due to non-commutativity of covariant derivatives), the Lagrangian will be gauge invariant provided:

$$
\begin{gathered}
\delta_{0} \mathcal{L}_{1}+\delta_{1} \mathcal{L}_{0}=0 \\
\delta_{0} \mathcal{L}_{0}+\delta_{1} \mathcal{L}_{1}+\delta_{0} \mathcal{L}_{2}+\delta_{2} \mathcal{L}_{0}=0 \\
\delta_{1} \mathcal{L}_{2}+\delta_{2} \mathcal{L}_{1}=0
\end{gathered}
$$

Following this general structure we introduce kinetic and mass terms for all fields involved:

$$
\begin{aligned}
\mathcal{L}_{0}= & \mathcal{L}_{0}\left(R_{\mu \nu}{ }^{\left(a_{k}\right),\left(b_{k}\right)}\right)+\sum_{n=0}^{k-1} \mathcal{L}_{0}\left(\Psi_{\mu \nu}{ }^{\left(a_{k}\right),\left(b_{n}\right)}\right)+\mathcal{L}_{0}\left(\Phi_{\mu}{ }^{\left(a_{k}\right)}\right) \\
\mathcal{L}_{2}= & \mathcal{L}_{2}\left(R_{\mu \nu}{ }^{\left(a_{k}\right),\left(b_{k}\right)}\right)+\sum_{n=1}^{k-1} \mathcal{L}_{2}\left(\Psi_{\mu \nu}{ }^{\left(a_{k}\right),\left(b_{n}\right)}\right)+\mathcal{L}_{2}\left(\Phi_{\mu}{ }^{\left(a_{k}\right)}\right)+ \\
& +b_{k, 1}\left\{\begin{array}{c}
\mu \nu \alpha \\
a b c
\end{array}\right\} \Psi_{\mu \nu}{ }^{a\left(a_{k-1}\right), b} \Phi_{\alpha}{ }^{c\left(a_{k-1}\right)}
\end{aligned}
$$

where all massless Lagrangians and mass terms (as well as initial gauge transformations $\delta_{0}$ and $\delta_{2}$ ) are exactly the same as in the previous section, but with ordinary derivatives replaced by the covariant ones. Note that there is a possibility of mixing between $Y(k+1,2)$ 
and $Y(k+1,0)$ in mass terms with the corresponding corrections for gauge transformations of the form:

$$
\begin{aligned}
& \delta_{2} \Omega_{\mu \nu}{ }^{\left(a_{k}\right), b c}= \frac{b_{k, 1}}{2(k+2)(d-3)(d-4)}\left[k e_{[\mu}{ }^{b} e_{\nu]}{ }^{c} \zeta^{\left(a_{k}\right)}-e_{[\mu}{ }^{b} e_{\nu]}{ }^{\left(a_{1}\right.} \zeta^{\left.a_{k-1}\right) c}+e_{[\mu}{ }^{c} e_{\nu]}{ }^{\left(a_{1}\right.} \zeta^{\left.a_{k-1}\right) b}+\right. \\
&+\frac{1}{(d+k-4)}\left[2 g^{\left(a_{1} a_{2}\right.} e_{[\mu}{ }^{[b} \zeta_{\nu]}{ }^{\left.\left.a_{k-2}\right) c\right]}+(k-1) g^{\left(a_{1}[b\right.} e_{\mu}{ }^{c]} \zeta_{\nu]}{ }^{\left.a_{k-1}\right)}-\right. \\
&\left.\left.\quad-g^{\left(a_{1}[b\right.} e_{[\mu}{ }^{a_{2}} \zeta_{\nu]}{ }^{\left.\left.a_{k-2}\right) c\right]}\right]\right] \\
& \delta_{2} \omega_{\mu}{ }^{a,\left(a_{k}\right)}=-b_{k, 1} \xi_{\mu}{ }^{\left(a_{k}\right), a}
\end{aligned}
$$

while the mass term for the $Y(k+1,1)$ field is absent.

Our next and the most important task - to construct a complete set of cross terms $\mathcal{L}_{1}$ as well as corresponding corrections for gauge transformations so that $\delta_{0} \mathcal{L}_{1}+\delta_{1} \mathcal{L}_{0}=0$. All our previous experience tells that it is enough to introduce such cross terms for the nearest neighbours only, i.e. main field with primary fields, primary with secondary and so on. Then the structure of general massive theory looks as shown on Figure 1, where each arrow corresponds to a cross term mixing two fields, while parameters $d_{k, n}$ will be determined below.

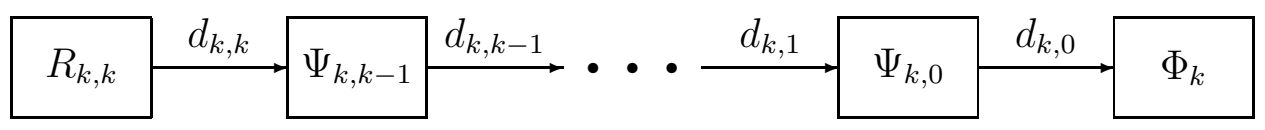

Figure 1: General massive $Y(k+1, k+1)$ theory

Let us consider all possible cross terms in turn.

$Y(k+1, n+1) \Leftrightarrow Y(k+1, n)$. Most of the cross terms belong to this class except the $n=1$ and $n=0$ cases which have to be considered separately. In this case additional terms for the Lagrangian look as follows:

$$
\begin{aligned}
& (-1)^{k+n} \mathcal{L}_{1}=d_{k, n}\left\{\begin{array}{l}
\mu \nu \alpha \beta \\
a b c d
\end{array}\right\}\left[\Omega_{\mu \nu}^{a\left(a_{k-1}\right), b\left(b_{n-1}\right), c} \Psi_{\alpha \beta} d\left(a_{k-1}\right),\left(b_{n-1}\right)+\right. \\
& \left.+\Omega_{\mu \nu}{ }^{a\left(a_{k-1}\right),\left(b_{n-1}\right), b} \Psi_{\alpha \beta}{ }^{c\left(a_{k-1}\right), d\left(b_{n-1}\right)}\right]
\end{aligned}
$$

In this, to compensate their non-invariance under the initial gauge transformations we have to introduce corresponding corrections for gauge transformations:

$$
\begin{aligned}
& \delta_{1} \Psi_{\mu \nu}{ }^{\left(a_{k}\right),\left(b_{n}\right)}=-\frac{d_{k, n}}{(k-n+2)(d+n-5)}\left[(k-n+1) \xi_{[\mu}{ }^{\left(a_{k}\right),\left(b_{n-1}\right.} e_{\nu]}^{\left.b_{1}\right)}-\right. \\
& \left.\left.-e_{[\nu}^{\left(a_{1}\right.} \xi_{\mu]} a_{k-1}\right)\left(b_{1}, b_{n-1}\right)+\ldots\right] \\
& \delta_{1} \Omega_{\mu \nu}{ }^{\left(a_{k}\right),\left(b_{n}\right), c}=\frac{d_{k, n}}{(k-n+2)(d+n-4)}\left[(k-n+1) e_{[\mu}{ }^{\left(b_{1}\right.} \eta_{\nu]}{ }^{\left.\left(a_{k}\right), b_{n-1}\right), c}-\right. \\
& \left.-e_{[\mu}^{\left(a_{1}\right.} \eta_{\nu]}^{\left.a_{k-1}\right)\left(b_{1}, b_{n-1}\right), c}+\ldots\right] \\
& \delta_{1} \Psi_{\mu \nu}\left(a_{k}\right),\left(b_{n-1}\right)=-\frac{(n-1)}{n} d_{k, n} \xi_{[\mu}^{\left(a_{k}\right),\left(b_{n-1}\right)}{ }_{\nu]} \\
& \delta_{1} \Omega_{\mu \nu}\left(a_{k}\right),\left(b_{n-1}\right), c=-d_{k, n}\left[\eta_{[\mu}\left(a_{k}\right),\left(b_{n-1}\right){ }_{\nu]}^{c}+\frac{1}{n} \eta_{[\mu}{ }^{\left(a_{k}\right),\left(b_{n-1}\right) c,}{ }_{\nu]}\right]
\end{aligned}
$$


where again dots stand for the terms which are necessary to make variations traceless and complete form of these expressions can be found in Appendix.

$Y(k+1,2) \Leftrightarrow Y(k+1,1)$ Here the additional terms for the Lagrangian has the following form:

$$
\begin{aligned}
(-1)^{k+1} \mathcal{L}_{1}= & d_{k, 1}\left\{\begin{array}{l}
\mu \nu \alpha \beta \\
a b c d
\end{array}\right\} \Omega_{\mu \nu}^{a\left(a_{k-1}\right), b c} \Psi_{\alpha \beta}{ }^{d\left(a_{k-1}\right)}+ \\
& +d_{k, 1}\left\{\begin{array}{l}
\mu \nu \alpha \\
a b c
\end{array}\right\}\left[\frac{1}{k} \Omega_{\mu}{ }^{\left(a_{k}\right), a b} \Psi_{\nu \alpha}^{\left(a_{k}\right), c}-2 \Omega_{\mu}{ }^{a\left(a_{k-1}\right), b e} \Psi_{\nu \alpha}{ }^{c\left(a_{k-1}\right), e}\right]
\end{aligned}
$$

while corresponding corrections for gauge transformations turn out to be:

$$
\begin{aligned}
& \delta_{1} \Psi_{\alpha \beta}{ }^{\left(a_{k}\right), b}=-\frac{d_{k, 1}}{(k+1)(d-4)}\left[k \xi_{[\alpha}{ }^{\left(a_{k}\right)} e_{\beta]}^{b}-e_{[\beta}{ }^{\left(a_{1}\right.} \xi_{\alpha]}{ }^{\left.a_{k-1}\right) b}+\right. \\
& \left.+\frac{1}{(d+k-3)}\left[2 g^{\left(a_{1} a_{2}\right.} \xi_{[\alpha, \beta]}^{\left.a_{k-2}\right) b}-(k-1) g^{b\left(a_{1}\right.} \xi_{[\alpha, \beta]}^{\left.a_{k-1}\right)}\right]\right] \\
& \delta_{1} \Omega_{\mu \nu}{ }^{\left(a_{k}\right), b c}=-\frac{k d_{k, 1}}{2(k+1)^{2}(d-4)}\left[(k+1) \eta^{\left(a_{k}\right),[b}{ }_{[\mu} e_{\nu]}^{c]}+e_{[\nu}^{\left(a_{1}\right.} \eta^{\left.a_{k-1}\right)[b, c]}{ }_{\mu]}+\right. \\
& \left.\left.\left.+\frac{1}{(d+k-4)}\left[2 g^{\left(a_{1} a_{2}\right.} \eta_{[\mu}^{\left.a_{k-2}\right)[b, c]} \nu\right]-k g^{\left[b \left(a_{1}\right.\right.} \eta_{[\mu}{ }^{\left.\left.a_{k-1}\right), c\right]} \nu\right]-2 g^{\left[b \left(a_{1}\right.\right.} \eta^{\left.\left.a_{k-1}\right) c\right]}{ }_{\mu \nu}\right]\right]+ \\
& +\frac{d_{k, 1}}{2(k+1)^{2}(d+k-3)}\left[(k+1) e_{[\nu}^{\left(a_{1}\right.} \eta_{\mu]}{ }^{\left.a_{k-1}\right), b c}-e_{[\nu}{ }^{\left(a_{1}\right.} \eta^{\left.a_{k-1}\right)[b, c]}{ }_{\mu]}-\right. \\
& \left.\left.\left.-\frac{1}{(d+k-4)}\left[2 g^{\left(a_{1} a_{2}\right.} \eta_{[\mu}^{\left.a_{k-2}\right)[b, c]} \nu\right]-k g^{\left[b \left(a_{1}\right.\right.} \eta_{[\mu}^{\left.\left.a_{k-1}\right), c\right]} \nu\right]-2 g^{\left[b \left(a_{1}\right.\right.} \eta^{\left.\left.a_{k-1}\right) c\right]}{ }_{\mu \nu}\right]\right] \\
& \delta_{1} \Psi_{\alpha \beta}{ }^{\left(a_{k}\right)}=-d_{k, 1} \xi_{[\alpha}{ }^{\left(a_{k}\right)}{ }_{\beta]}, \quad \delta_{1} \Omega_{\mu}{ }^{\left(a_{k}\right), b c}=-2 d_{k, 1} \eta_{\mu}{ }^{\left(a_{k}\right), b c}
\end{aligned}
$$

Note that in this (and only this) case there exist two independent ways to obtain correct tensor structure for $\Omega_{\mu \nu}\left(a_{k}\right), b c$ transformations 1 .

$Y(k+1,1) \Leftrightarrow Y(k+1,0)$ These terms have already been considered in [1], but we reproduce them here in our current notations:

$$
(-1)^{k} \mathcal{L}_{1}=d_{k, 0}\left[\frac{1}{k}\left\{\begin{array}{l}
\mu \nu \\
a b
\end{array}\right\} \Omega_{\mu}^{\left(a_{k}\right), a b} \Phi_{\nu}{ }^{\left(a_{k}\right)}+\left\{\begin{array}{l}
\mu \nu \alpha \\
a b c
\end{array}\right\} \omega_{\mu}^{a, b\left(a_{k-1}\right)} \Psi_{\nu \alpha}{ }^{c\left(a_{k-1}\right)}\right]
$$

together with appropriate corrections for gauge transformations:

$$
\begin{aligned}
\delta_{1} \Psi_{\mu \nu}{ }^{\left(a_{k}\right)}= & -\frac{d_{k, 0}}{2(k+2)(d+k-4)} e_{[\mu}^{\left(a_{1} \zeta_{\nu]}{ }^{\left.a_{k-1}\right)}\right.} \\
\delta_{1} \Omega_{\mu}{ }^{\left(a_{k}\right), b c}= & -\frac{d_{k, 0}}{2(k+2)(d-3)}\left[(k+1) e_{\mu}{ }^{[b} \chi^{c],\left(a_{k}\right)}+e_{\mu}{ }^{\left(a_{1}\right.} \chi^{\left.[b, c] a_{k-1}\right)}+\right. \\
& \left.-\frac{1}{(d+k-4)}\left[2 g^{\left(a_{1} a_{2}\right.} \chi^{\left.[b, c] a_{k-2}\right)}{ }_{\mu}+g^{\left(a_{1}[b\right.} \chi_{\mu}{ }^{\left.c] a_{k-1}\right)}+k g^{\left(a_{1}[b\right.} \chi^{\left.c], a_{k-1}\right)}{ }_{\mu}\right]\right] \\
\delta_{1} \Phi_{\mu}{ }^{\left(a_{k}\right)}= & -d_{k, 0} \xi_{\mu}^{\left(a_{k}\right)}, \quad \delta_{1} \omega_{\mu}^{a,\left(a_{k}\right)}=-\frac{d_{k, 0}}{2} \eta^{\left(a_{k}\right), a}{ }_{\mu}
\end{aligned}
$$

At this point we have a whole Lagrangian (i.e. a complete set of kinetic, cross and mass terms) as well as complete set of gauge transformations. In this, all parameters in

\footnotetext{
${ }^{1}$ Author is grateful to E. D. Skvortsov for pointing this out
} 
gauge transformations are expressed in terms of the Lagrangian ones $d_{k, n}$ and $a_{k, n}$ so that all variations of order $m$ cancel $\delta_{0} \mathcal{L}_{1}+\delta_{1} \mathcal{L}_{0}=0$. Thus our next task - to consider all variations of order $m^{2}$ and $m^{3}$ and require their cancellation. We will not give here these lengthy but straightforward calculations and reproduce the final results only.

Recall that working with gauge invariant formulation for massive fields it is natural to define massless limit as the one where all additional Goldstone fields decouple from the main one. In the case at hands this corresponds to the limit $d_{k, k} \rightarrow 0$ and indeed in a flat Minkowski space this parameter would be proportional to the mass. As we have already mentioned in the Introduction, we will not try to give any strict definition of what is mass in (A) $d S$ spaces. Instead, we will simply use this parameter for the investigation of all special limits that exist in such spaces. Let us denote $M^{2}=(k-1) d_{k, k}^{2}$. Then for the parameter $a_{k, k}$ determining mass term for the main field we obtain:

$$
a_{k, k}=-\frac{(k+2)}{4 k}\left[M^{2}-2 k(d+k-5) \kappa\right]
$$

Moreover, all other mass terms turn out to be proportional to the main one:

$$
a_{k, n}=\frac{k(d+k-4)}{n(d+n-4)} a_{k, k}, \quad n \geq 1
$$

the only exceptions being:

$$
b_{k, 1}=d_{k, 1} d_{k, 0}, \quad b_{k}=\frac{(d-2)}{(d-4)} d_{k, 1}^{2}
$$

Also we obtain a number of recurrent relations on the parameters $d_{k, n}$ which can be easily solved and give us:

$$
\begin{gathered}
d_{k, n}^{2}=\frac{(k-n+2)(d+k+n-4)}{2(n-1)(d+2 n-4)}\left[M^{2}-2(k-n)(d+k+n-5) \kappa\right], \quad n \geq 2 \\
d_{k, 1}^{2}=\frac{(k+1)(d+k-3)}{2(d-2)}\left[M^{2}-2(k-1)(d+k-4) \kappa\right] \\
d_{k, 0}^{2}=\frac{(k+2)(d+k-4)}{(d-4)}\left[M^{2}-2 k(d+k-5) \kappa\right]
\end{gathered}
$$

Thus all the parameters in the Lagrangian and gauge transformations are expressed in terms of one main parameter $M$ and we are ready to analyze the results obtained.

Let us begin with $A d S$ space. As we have already noted in the previous section, massless tensor $Y(k+1, k+1)$ admits a deformation into $A d S$ space without introduction of any additional fields. And indeed, from the formulas given above we see that nothing prevent us from considering massless limit $M \rightarrow 0$ where all additional fields decouple from the main one as shown on Figure 2. In this, the remaining fields describe partially massless theory for the $Y(k+1, k)$ tensor (see the discussion of such theories in the next section).

Let us turn to the $d S$ space. First of all, from the expressions for the parameters $d_{k, n}$ we see that there is a unitary forbidden region $M^{2}<2 k(d+k-5) \kappa$. At the boundary of this region we obtain first partially massless theory where the last field $Y(k+1,0)$ decouples 


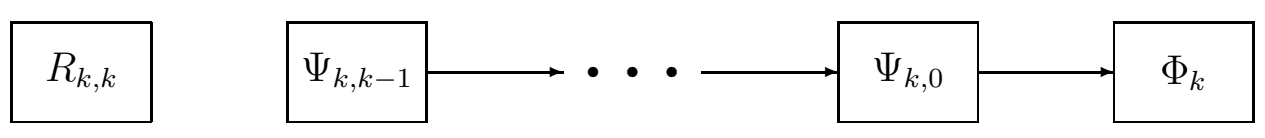

Figure 2: Massless limit in AdS space

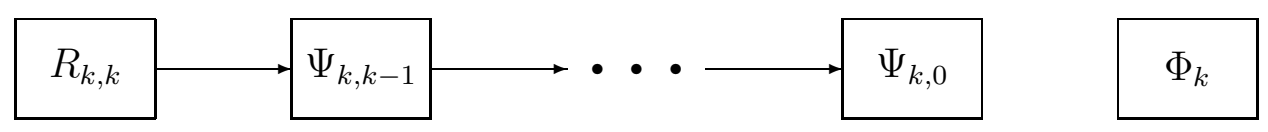

Figure 3: Unitary partially massless limit in $d S$ space

from the rest ones as shown on Figure 3. Note that at this point all mass parameters $a_{k, n}$ become equal to zero. Inside the forbidden region we find a number of partially massless limits which happens each time then one of the parameters $d_{k, n}$ becomes equal to zero. In this case the whole system decompose into two disconnected subsystems as shown on Figure 4. Both subsystems describe partially massless theories (for the discussion of the second one

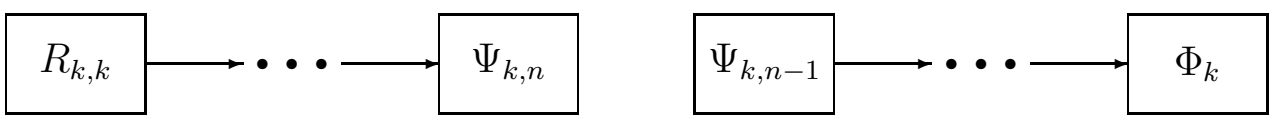

Figure 4: Example of non-unitary partially massless limit in $d S$ space

see next section) however in this case we have $d_{k, m}^{2}>0$ for $m>n$ while $d_{k, m}^{2}<0$ for $m<n$.

\section{Massive tensor $Y(k+1, l+1)$}

In this section we consider gauge invariant formulation for massive $Y(k+1, l+1)$ tensor with $k \neq l$. This case will be more complicated but our general strategy will be the same. First of all we have to find a set of additional fields necessary for such description. Using the fact that in general tensor $Y(m+1, n+1)$ has two gauge transformations with parameters corresponding to $Y(m+1, n)$ and $Y(m, n+1)$ and taking into account reducibility of these transformations with parameter $Y(m, n)$, it is not hard to check that we need $Y(m+1, n)$ with $l \leq m \leq k, 0 \leq n \leq l+1$. All these fields as well as parameters determining cross terms (see below) are shown on Figure 5 .

First of all we introduce kinetic and mass terms for all fields involved:

$$
\begin{gathered}
\mathcal{L}_{0}=\sum_{m=l}^{k} \sum_{n=0}^{l} \mathcal{L}_{0}\left(\Psi_{\mu \nu}{ }^{\left(a_{m}\right),\left(b_{n}\right)}\right)+\sum_{m=l}^{k} \mathcal{L}_{0}\left(\Phi_{\mu}{ }^{\left(a_{m}\right)}\right) \\
\mathcal{L}_{2}=\sum_{m=l}^{k} \sum_{n=0}^{l} \mathcal{L}_{2}\left(\Psi_{\mu \nu}{ }^{\left(a_{m}\right),\left(b_{n}\right)}\right)+\sum_{m=l}^{k}\left[b_{m, 1}\left\{\begin{array}{c}
\mu \nu \alpha \\
a b c
\end{array}\right\} \Psi_{\mu \nu}{ }^{a\left(a_{m-1}\right), b} \Phi_{\alpha}{ }^{c\left(a_{m-1}\right)}+\mathcal{L}_{2}\left(\Phi_{\mu}{ }^{\left(a_{m}\right)}\right)\right]
\end{gathered}
$$




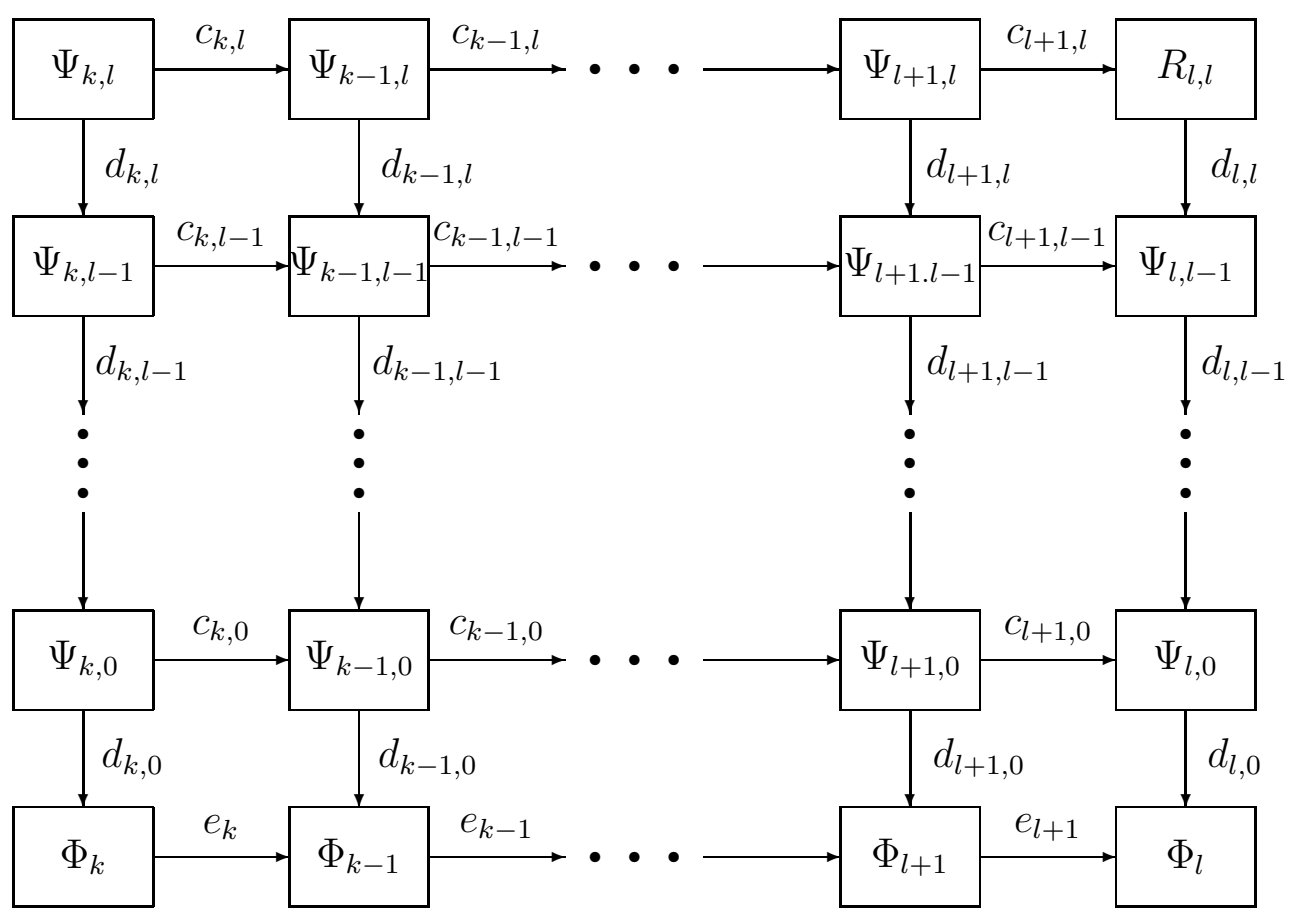

Figure 5: General massive $Y(k+1, l+1)$ theory

where all kinetic and mass terms (as well as initial gauge transformations $\delta_{0}$ and $\delta_{2}$ ) are the same as in Section 1 but with the ordinary derivatives replaced by the covariant ones and we again take into account the possibility of mixing for $Y(m+1,2)$ and $Y(m+1,0)$ tensors.

Our next and important task - to determine a set of cross terms mixing these fields together as well as corresponding corrections for gauge transformations. As in all previously considered cases it is enough to introduce such terms for the nearest neighbours only. For the case at hands it means that general $Y(m+1, n+1)$ tensor will have cross terms with four nearest fields only as shown on Figure 6. The cross terms corresponding to vertical arrows with parameters $d_{m, n}$ have already been considered in the previous section so we will not repeat them here. Let us consider cross terms corresponding to horizontal arrows with parameters $c_{m, n}$.

$Y(m+1, n+1) \Leftrightarrow Y(m, n+1)$ In this case additional terms for the Lagrangian can be written as follows:

$$
\begin{aligned}
(-1)^{m+n} \mathcal{L}_{1}=c_{m, n}\left\{\begin{array}{c}
\mu \nu \alpha \beta \\
a b c d
\end{array}\right\} & {\left[\Omega_{\mu \nu}^{a\left(a_{m-1}\right), b\left(b_{n-1}\right), c} \Psi_{\alpha \beta}{ }^{\left(a_{m-1}\right), d\left(b_{n-1}\right)}+\right.} \\
& \left.+\Omega_{\mu \nu}{ }^{\left(a_{m-1}\right), a\left(b_{n-1}\right), b} \Psi_{\alpha \beta}{ }^{c\left(a_{m-1}\right), d\left(b_{n-1}\right)}\right]
\end{aligned}
$$

while corresponding corrections for gauge transformations which are necessary to compensate their non-invariance under the initial gauge transformations look like (for complete expressions see Appendix):

$$
\delta_{1} \Psi_{\mu \nu}^{\left(a_{m}\right),\left(b_{n}\right)}=-\frac{c_{m, n}}{(d+m-4)}\left[e_{[\mu}^{\left(a_{1}\right.} \xi_{\nu]}^{\left.a_{m-1}\right),\left(b_{n}\right)}+\ldots\right]
$$




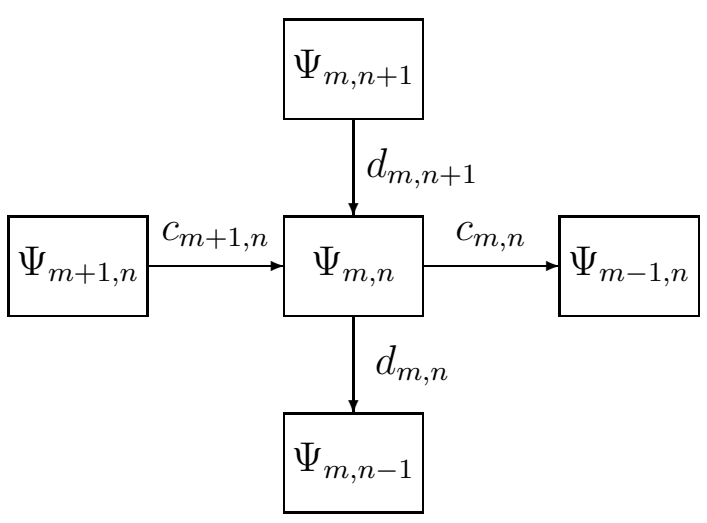

Figure 6: Illustration on possible cross terms

$$
\begin{aligned}
\delta_{1} \Omega_{\mu \nu}{ }^{\left(a_{m}\right),\left(b_{n}\right), c}= & -\frac{c_{m, n}}{(d+m-3)}\left[e_{[\mu}{ }^{\left(a_{1}\right.} \eta_{\nu]}{ }^{\left.a_{m-1}\right),\left(b_{n}\right), c}+\ldots\right] \\
\delta_{1} \Psi_{\mu \nu}{ }^{\left(a_{m-1}\right),\left(b_{n}\right)}= & \frac{(m-1) c_{m, n}}{(m+1)}\left[\xi_{[\mu, \nu]}{ }^{\left(a_{m-1}\right),\left(b_{n}\right)}+\frac{1}{(m-n+1)} \xi_{[\mu}{ }^{\left(a_{m-1}\right)\left(b_{1}, b_{n-1}\right)}{ }_{\nu]}\right] \\
\delta_{1} \Omega_{\mu \nu}{ }^{\left(a_{m-1}\right),\left(b_{n}\right), c}= & \frac{(m-1) c_{m, n}}{m}\left[\eta_{[\mu, \nu]}{ }^{\left(a_{m-1}\right),\left(b_{n}\right), c}+\frac{1}{(m-n+1)} \eta_{[\mu}{ }^{\left(a_{m-1}\right)\left(b_{1}, b_{m-1}\right)}{ }_{\nu]}^{c}+\right. \\
& \left.\quad+\frac{1}{(m+1)} \eta_{[\mu}{ }^{\left(a_{m-1}\right) c,\left(b_{n}\right)}{ }_{\nu]}+\frac{1}{(m+1)(m-n+1)} \eta_{[\mu}{ }^{\left(a_{m-1}\right)\left(b_{1}, b_{n-1}\right) c}{ }_{\nu]}\right]
\end{aligned}
$$

Once again there are two cases that have to be considered separately.

$Y(m+1,1) \Leftrightarrow Y(m, 1)$ Here cross terms have the form:

$$
(-1)^{m} \mathcal{L}_{1}=c_{m, 0}\left\{\begin{array}{c}
\mu \nu \alpha \\
a b c
\end{array}\right\}\left[\Omega_{\mu}{ }^{a\left(a_{m-1}\right), b c} \Psi_{\nu \alpha}{ }^{\left(a_{m-1}\right)}+\Omega_{\mu}{ }^{\left(a_{m-1}\right), a b} \Psi_{\nu \alpha}{ }^{c\left(a_{m-1}\right)}\right]
$$

while corresponding corrections for gauge transformations turn out to be:

$$
\begin{aligned}
& \delta_{1} \Psi_{\mu \nu}{ }^{\left(a_{m}\right)}=-\frac{c_{m, 0}}{(d+m-4)}\left[e_{[\mu}{ }^{\left(a_{1}\right.} \xi_{\nu]}^{\left.a_{m-1}\right)}+\frac{2}{(d+2 m-4)} g^{\left(a_{1} a_{2}\right.} \xi_{[\mu, \nu]}{ }^{\left.a_{m-2}\right)}\right] \\
& \delta_{1} \Omega_{\mu}{ }^{\left(a_{m}\right), a b}=-\frac{c_{m, 0}}{(d+m-2)}\left[e_{\mu}{ }^{\left(a_{1}\right.} \eta^{\left.a_{m-1}\right), a b}-\frac{2}{(d+2 m-4)} g^{\left(a_{1} a_{2}\right.} \eta_{\mu}{ }^{\left.a_{m-2}\right), a b}-\right. \\
&\left.-\frac{2}{(d+m-4)(d+2 m-4)} g^{\left(a_{1} a_{2}\right.} \eta^{\left.a_{m-2}\right)[a, b]}+\frac{1}{(d+m-4)} g^{\left(a_{1}[a\right.} \eta^{\left.\left.a_{m-1}\right), b\right]}\right] \\
& \delta_{1} \Psi_{\mu \nu}{ }^{\left(a_{m-1}\right)}= \frac{(m-1) c_{m, 0}}{(m+1)} \xi_{[\mu, \nu]}{ }^{\left(a_{m-1}\right)} \\
& \delta_{1} \Omega_{\mu}{ }^{\left(a_{m-1}\right), a b}=-c_{m, 0}\left[\eta_{\mu}{ }^{\left(a_{m-1}\right), a b}-\frac{1}{(m+1)} \eta^{\left(a_{m-1}\right)[a, b]}{ }_{\mu}\right] \\
& Y(m+1,0) \Leftrightarrow Y(m, 0) \text { At last we need the following cross terms: } \\
&(-1)^{m} \mathcal{L}_{1}=e_{m}\left\{\begin{array}{l}
\mu \nu \\
a b
\end{array}\right\}\left[\omega_{\mu}{ }^{a, b\left(a_{m-1}\right)} \Phi_{\nu}{ }^{\left(a_{m-1}\right)}+\omega_{\mu}{ }^{a,\left(a_{m-1}\right)} \Phi_{\nu}{ }^{b\left(a_{m-1}\right)}\right]
\end{aligned}
$$


and corresponding corrections for gauge transformations:

$$
\begin{aligned}
\delta_{1} \Phi_{\mu}{ }^{\left(a_{m}\right)}= & -\frac{e_{m}}{2(d+m-3)}\left[e_{\mu}{ }^{\left(a_{1}\right.} \zeta^{\left.a_{m-1}\right)}-\frac{2}{(d+2 m-4)} g^{\left(a_{1} a_{2}\right.} \zeta_{\mu}^{\left.a_{m-2}\right)}\right] \\
\delta_{1} \omega_{\mu}{ }^{a,\left(a_{m}\right)}= & -\frac{e_{m}}{2(d+m-2)}\left[\chi^{a,\left(a_{m-1}\right.} e_{\mu}^{\left.a_{1}\right)}-\frac{2}{(d+2 m-4)} \chi^{a,\left(a_{k-2}\right.}{ }_{\mu} g^{\left.a_{1} a_{2}\right)}+\right. \\
& \left.+\frac{2}{(d+m-3)(d+2 m-4)} g^{\left(a_{1} a_{2}\right.} \chi_{\mu}^{\left.a_{m-2}\right) a}-\frac{1}{(d+m-3)} g^{a\left(a_{1}\right.} \chi_{\mu}{ }^{\left.a_{m-1}\right)}\right] \\
\delta_{1} \Phi_{\mu}{ }^{\left(a_{m-1}\right)}= & -\frac{(m-1) e_{m}}{2 m} \zeta_{\mu}{ }^{\left(a_{m-1}\right)} \\
\delta_{1} \omega_{\mu}{ }^{a,\left(a_{m-1}\right)}= & -\frac{e_{m}}{2}\left[\chi^{a,\left(a_{m-1}\right)}{ }_{\mu}+\frac{1}{m} \chi_{\mu}{ }^{a\left(a_{m-1}\right)}\right]
\end{aligned}
$$

At this point we have a whole Lagrangian (i.e. a complete set of kinetic, cross and mass terms) as well as complete set of gauge transformations. In this, all parameters in gauge transformations are expressed in terms of the Lagrangian ones $d_{m, n}, c_{m, n}$ and $a_{m, n}$ so that all variations of order $m$ cancel $\delta_{0} \mathcal{L}_{1}+\delta_{1} \mathcal{L}_{0}=0$. Thus our next task - to consider all variations of order $m^{2}$ and $m^{3}$ and require their cancellation. Again we will not give here these very lengthy but straightforward calculations and reproduce our final results only.

Our main tensor $Y(k+1, l+1)$ has two gauge transformations and as a result there are two parameters $d_{k, l}$ and $c_{k, l}$ determining its mixing with two primary fields $Y(k+1, l)$ and $Y(k, l+1)$. According to our usual definition massless limit requires that both $d_{k, l} \rightarrow 0$ and $c_{k, l} \rightarrow 0$ simultaneously. But this is possible in a flat Minkowski space only (where both parameters are proportional to mass). Again we will not insist on any definition of what is mass in $(A) d S$ spaces and simply use one of these parameters as our main one. Let us introduce a notation $M^{2}=\frac{(k-l+1)(l-1)}{(k-l+2)} d_{k, l}^{2}$. Then we obtain a following expression for the parameter $a_{k, l}$ determining a mass term for the main field:

$$
a_{k, l}=-\frac{(k+2)(l+1)}{2 l(k+1)}\left[M^{2}-l(d+l-5) \kappa\right]
$$

Moreover, all other mass terms turn out to be proportional to this main one:

$$
a_{m, n}=\frac{l(k+1)(d+k-3)(d+l-4)}{n(m+1)(d+m-3)(d+n-4)} a_{k, l}
$$

the only exceptions being:

$$
b_{m, 1}=d_{m, 1} d_{m, 0}, \quad b_{m}=\frac{(d-2)}{(d-4)} d_{m, 1}^{2}
$$

Further we obtain the following expression for parameters $c_{m, l}$ corresponding to the topmost row on Figure 5:

$$
c_{m, l}^{2}=\frac{(k-m+1)(d+k+m-2)}{(m-1)(d+2 m-2)}\left[M^{2}+(m-l+1)(d+m+l-4) \kappa\right]
$$

as well as parameters $d_{k, n}$ corresponding to the leftmost column:

$$
d_{k, n}^{2}=\frac{(l-n+1)(k-n+2)(d+l+n-4)}{(k-n+1)(n-1)(d+2 n-4)}\left[M^{2}-(l-n)(d+n+l-5) \kappa\right], \quad n \geq 2
$$




$$
\begin{aligned}
d_{k, 1}^{2} & =\frac{l(k+1)(d+l-3)}{k(d-2)}\left[M^{2}-(l-1)(d+l-4) \kappa\right] \\
d_{k, 0}^{2} & =\frac{2(k+2)(l+1)(d+l-4)}{(k+1)(d-4)}\left[M^{2}-l(d+l-5) \kappa\right]
\end{aligned}
$$

It is very important (and this gives a nice check for all lengthy calculations) that all parameters $d_{m, n}$ corresponding to the same row on Figure 5 turn out to be proportional to the leftmost one $d_{k, n}$ :

$$
d_{m, n}^{2}=\frac{(k-n+1)(d+k+n-3)}{(m-n+1)(d+m+n-3)} d_{k, n}^{2}
$$

Similarly, all parameters $c_{m, n}$ and $e_{m}$ corresponding to the same column turn out to be proportional to the topmost one $c_{m, l}$ :

$$
\begin{gathered}
c_{m, n}^{2}=\frac{(m-l)(d+m+l-3)}{(m-n)(d+m+n-3)} c_{m, l}^{2} \\
e_{m}^{2}=\frac{4(m-l)(d+m+l-3)}{(m+1)(d+m-4)} c_{m, l}^{2}
\end{gathered}
$$

Thus all the parameters in the Lagrangian and gauge transformations are expressed in terms of one main parameter $M$ and we are ready to investigate all special limits. Let us start with $A d S$ space $(\kappa<0)$. From the expression for the parameters $c_{m, l}$ above we see that in this case we have unitary forbidden region $M^{2}<-(k-l+1)(d+k+l-4) \kappa$. At the boundary of this region we find a most interesting (and the only unitary) limit when $c_{k, l}$ (and hence all $c_{m, l}$ and $e_{l}$ ) becomes equal to zero. In this case the whole system decomposes into two disconnected subsystems as shown on Figure 7 . A set of fields in the leftmost

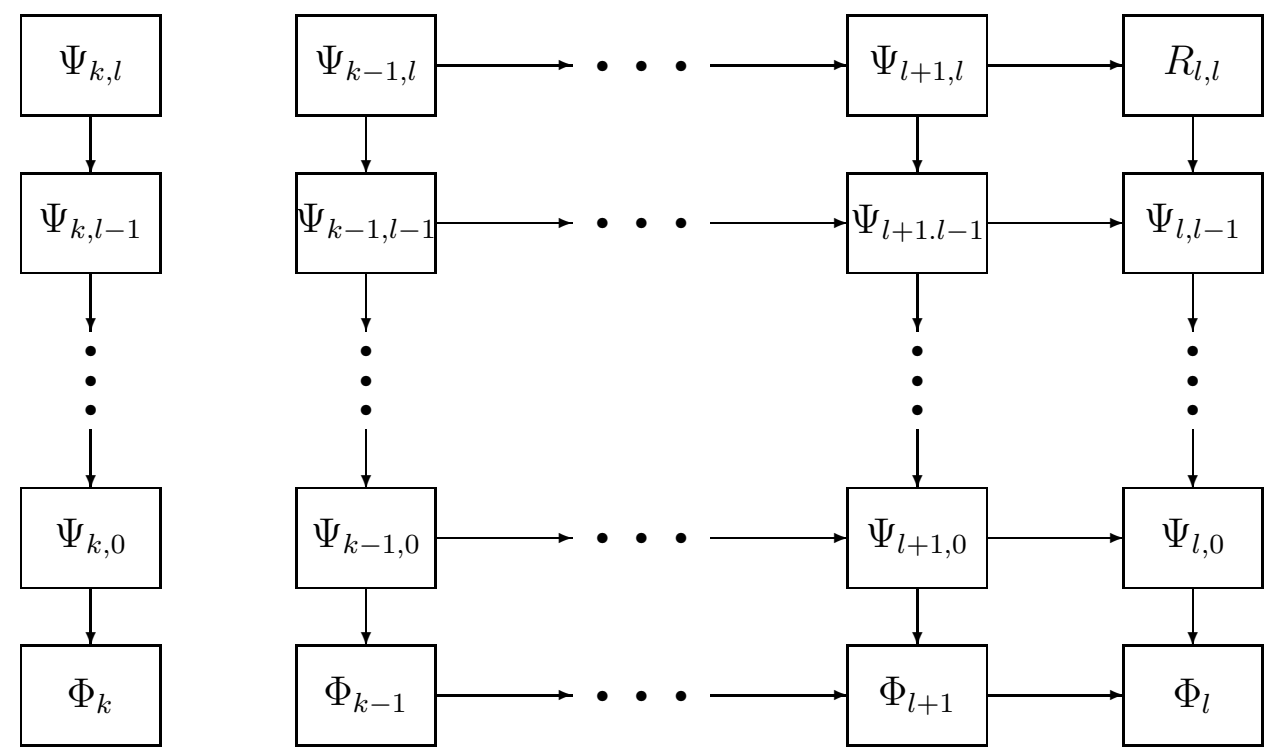

Figure 7: Unitary (partially) massless limit in $A d S$ space

column describes partially massless theory for the $Y(k+1, l+1)$ tensor. Note that from the 
anti-de Sitter group point of view it corresponds to irreducible representation with minimum number of degrees of freedom so such theory can be called massless though in a flat limit it decomposes into a number of massless representations of Lorentz group. Any way, this shows a minimum number of additional fields which are necessary to deform massless $Y(k+1, l+1)$ theory into $A d S$ space. The pattern corresponds to cutting the boxes from the second row of Young tableau until we end up with diagram having one row only in complete agreement with general discussion in [10. At the same time the rest fields describe massive theory for $Y(k, l+1)$ tensor.

Inside the unitary forbidden region we find a number of non-unitary partially massless limits. They arise each time when one of the $c_{m, l}$ (and hence all the parameters $c_{m, n}$ and $e_{m}$ ) becomes equal to zero. In this case the whole system also decomposes into two disconnected subsystems as shown on Figure 8. Fields in the left block on Figure 8 describes partially

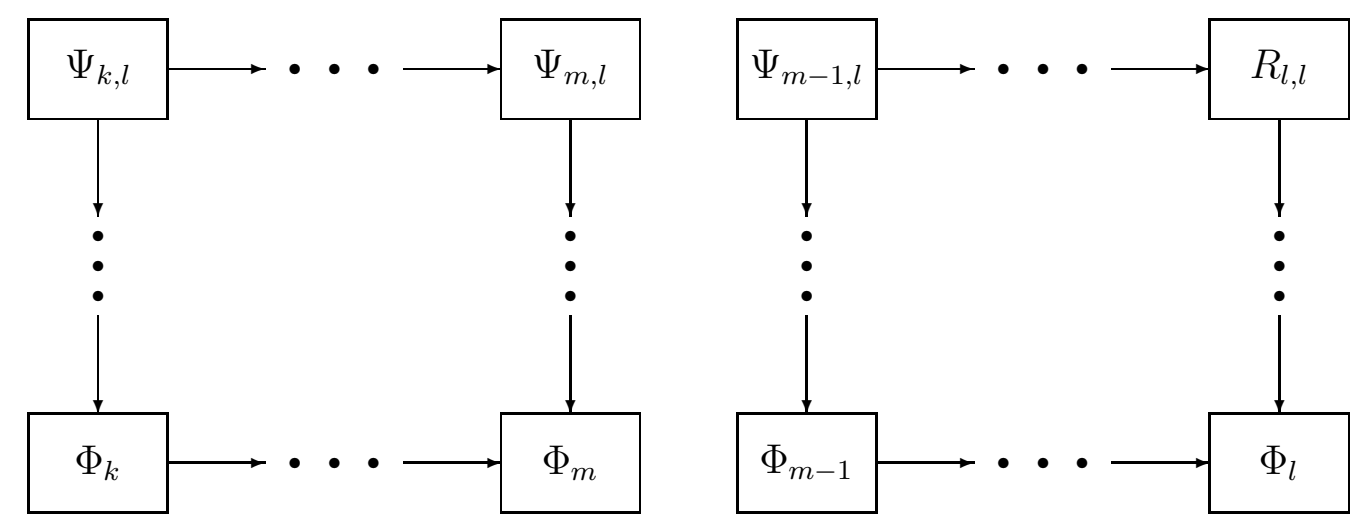

Figure 8: Example of non-unitary partially massless limit in $A d S$ space

massless theory (this time really partially massless even from the anti-de Sitter group point of view), while fields in the right block give massive theory for $Y(m, l+1)$ tensor. Note however that in this case we have $c_{p, l}^{2}<0$ for all $m<p \leq k$.

Let us turn to the $d S$ space $(\kappa>0)$. From the expression for the parameters $d_{k, n}$ above we see that in this case we also have unitary forbidden region $M^{2}<l(d+l-5) \kappa$. At the boundary of this region we have $d_{k, 0}=0$ (and hence all $d_{m, 0}=0$ ) and we find a first partially massless limit. Here the whole system also decomposes into two disconnected subsystems (but this time diagram splits vertically) as shown on Figure 9. Contrary to what we have seen in $A d S$ space both subsystems describe partially massless theories for $Y(k+1, l+1)$ and $Y(k+1,0)$ tensors correspondingly.

Inside the forbidden region we find a number of partially massless limits. The most interesting one arises when $d_{k, l}=0$ (and hence all $d_{m, l}=0$ ). In this case fields in the upper row decouple from the rest ones as shown on Figure 10. These fields describe a theory corresponding to irreducible representation of de Sitter group with minimal number of degrees of freedom, so from the de Sitter group point of view it can be called massless. But in a flat limit it decomposes into a number of massless representations of Lorentz group, the pattern now corresponds to cutting boxes from the first row of Young tableau until we end up with diagram having the same number of boxes in both rows. The rest fields give 


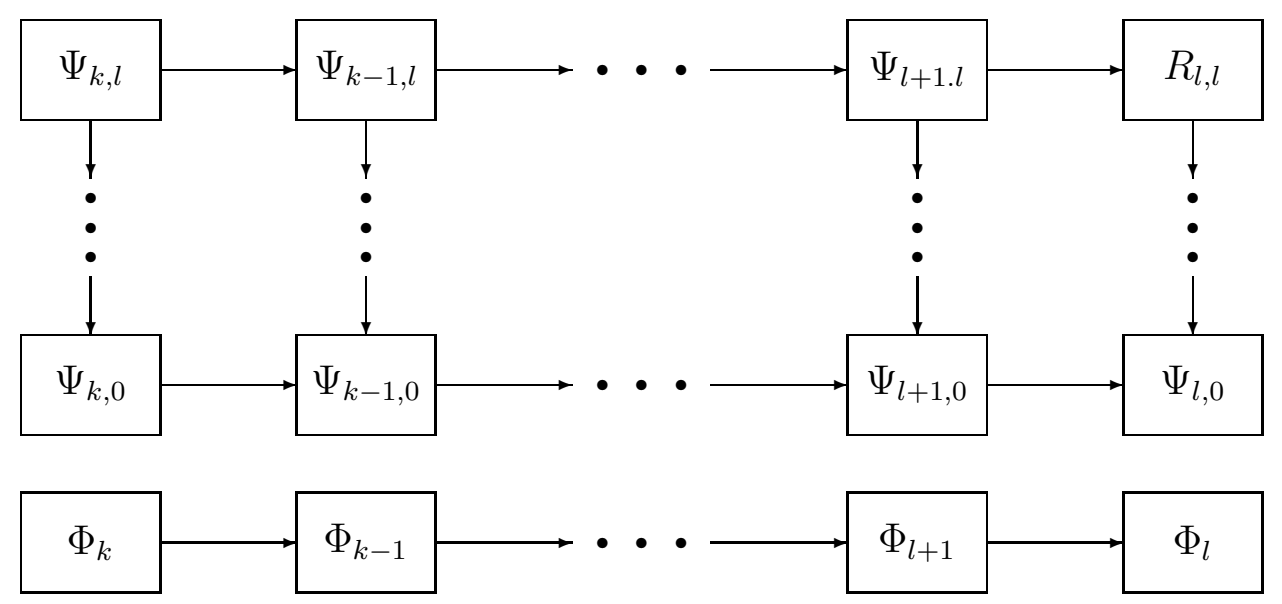

Figure 9: Unitary partially massless limit in $d S$ space

partially massless theory for $Y(k+1, l)$ tensor. The reason is that for description of massive theory we must have one more column of fields as also shown on Figure 10. Note however that in this case we obtain $d_{k, n}^{2}<0$ for all $n<l$ so this theory is non-unitary.

Besides these two cases we find a number of partially massless limits which arise each time when one of the parameters $d_{k, n}$ (and hence all parameters $d_{m, n}$ ) becomes equals to zero. At this point the whole system also decomposes into two disconnected subsystems (and diagram splits vertically into two blocks) as shown on Figure 11. The upper block gives one more example of partially massless theory for the $Y(k+1, l+1)$ tensor, while the lower block describes non-unitary (because $d_{k, p}^{2}<0$ for all $p<n$ ) partially massless theory for the $Y(k+1, n)$ tensor we are already familiar with. Indeed, to describe the massive theory for this tensor we need one more block of fields also shown on Figure 11. In this, the same partially massless theory can be obtained as a limit for massive $Y(k+1, l+1)$ theory as well as a limit for massive $Y(k+1, n)$ theory showing a consistency for the whole construction.

\section{Conclusion}

Thus we have complete frame-like gauge invariant formulation both for bosonic mixed symmetry tensors as well as fermionic mixed symmetry spin-tensors corresponding to arbitrary Young tableau with two rows. In all cases considered the same set of fields allows one to describe massive theory in Minkowski space and (anti-)de Sitter spaces with arbitrary cosmological constant. It is worth to note a great similarity of results for bosonic and fermionic cases [25]. The main difference (the same as for completely symmetric (spin-)tensors [23]) is that in bosonic case we find first partially limits at the boundaries of unitary forbidden regions while for fermions all partially massless theories "live" inside the forbidden regions.

\section{Acknowledgment}

Author is grateful to E. D. Skvortsov for stimulating discussions and correspondence. 


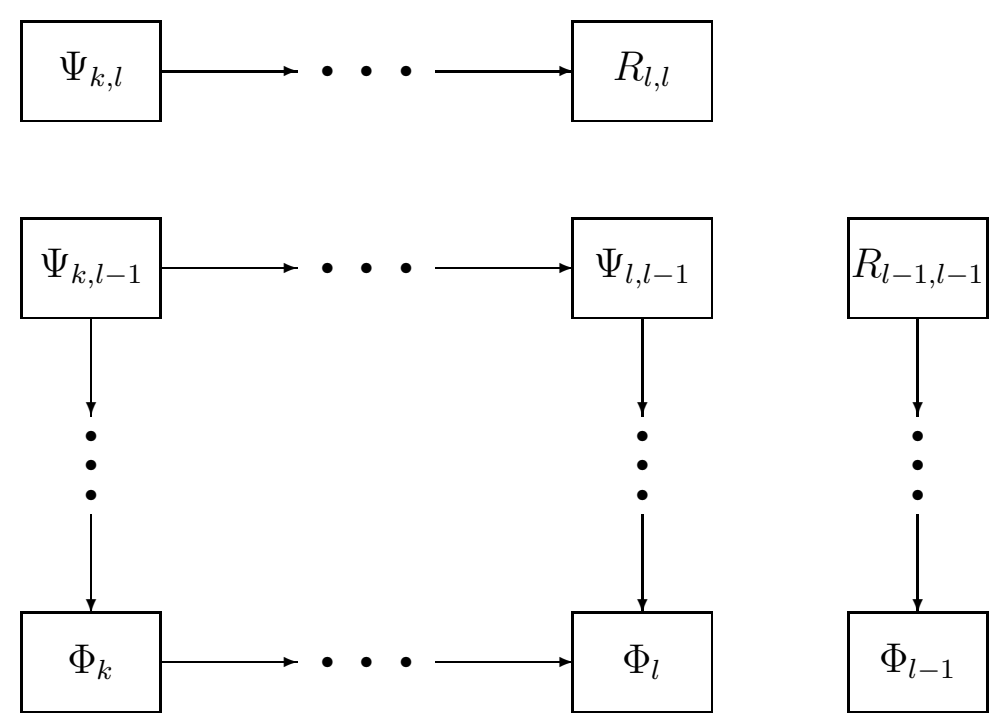

Figure 10: (Partially) massless limit in $d S$ space

\section{A Explicit expressions for some complicated formulas}

Complete form of Formula (14) in Section 1:

$$
\begin{aligned}
& \delta_{2} \Omega_{\mu \nu}\left(a_{k}\right),\left(b_{l}\right), c=-\frac{2 a_{k, l}}{(k+2)(l+1)(d-4)}\left[(k+1) l e_{[\mu}{ }^{c} \xi_{\nu]}{ }^{\left(a_{k}\right),\left(b_{l}\right)}-l e_{[\mu}{ }^{\left(a_{1}\right.} \xi_{\nu]}{ }^{\left.a_{k-1}\right) c,\left(b_{l}\right)}+\right. \\
& +e_{[\mu}^{\left(a_{1}\right.} \xi_{\nu]}^{\left.a_{k-1}\right)\left(b_{1}, b_{l-1}\right) c}-(k+1) e_{[\mu}{ }^{\left(b_{1}\right.} \xi_{\nu]}{ }^{\left.\left(a_{k}\right), b_{l-1}\right) c}+ \\
& +\rho_{1} g^{\left(a_{1} a_{2}\right.} \xi_{[\mu, \nu]}^{\left.a_{k-2}\right)\left(b_{1}, b_{l-1}\right) c}+\rho_{2} g^{\left(a_{1} a_{2}\right.} \xi_{[\mu, \nu]}^{\left.a_{k-2}\right) c,\left(b_{l}\right)}+ \\
& +\rho_{3} g^{\left(a_{1} a_{2}\right.} \xi_{[\mu}^{\left.a_{k-2}\right)\left(b_{1} b_{2}, b_{l-2}\right) c}{ }_{\nu]}+\rho_{4} g^{\left(a_{1} a_{2}\right.} \xi_{[\mu}^{\left.a_{k-2}\right) c\left(b_{1}, b_{l-1}\right)}{ }_{\nu]}+ \\
& +\rho_{5} g^{\left(a _ { 1 } \left(b_{1}\right.\right.} \xi_{[\mu, \nu]}^{\left.\left.a_{k-1}\right), b_{l-1}\right) c}+\rho_{6} g^{\left(a _ { 1 } \left(b_{1}\right.\right.} \xi_{[\mu}^{\left.\left.a_{k-1}\right) b_{2}, b_{l-2}\right) c}{ }_{\nu]}+ \\
& +\rho_{7} g^{\left(a _ { 1 } \left(b_{1}\right.\right.} \xi_{[\mu}^{\left.\left.a_{k-1}\right) c, b_{l-1}\right)}{ }_{\nu]}+\rho_{8} g^{\left(b_{1} b_{2}\right.} \xi_{[\mu}^{\left.\left(a_{k}\right), b_{l-2}\right) c}{ }_{\nu]}+
\end{aligned}
$$

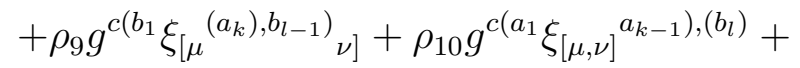

$$
\begin{aligned}
& \left.+\rho_{11} g^{c\left(a_{1}\right.} \xi_{[\mu}^{\left.a_{k-1}\right)\left(b_{1}, b_{l-1}\right)}{ }_{\nu]}\right] \\
& \rho_{1}=\frac{2}{(d+k-4)}, \quad \rho_{2}=-\frac{2 l}{(d+k-4)}, \quad \rho_{3}=-\frac{4}{(d+k-4)(d+l-5)} \\
& \rho_{4}=\frac{2(l-1)}{(d+k-4)(d+l-5)}, \quad \rho_{5}=-\frac{k}{(d+k-4)}, \quad \rho_{6}=\frac{(d+2 k-4)}{(d+k-4)(d+l-5)} \\
& \rho_{7}=-\frac{[l(d+k-4)-k]}{(d+k-4)(d+l-5)}, \quad \rho_{8}=-\frac{2(k+1)}{(d+l-5)}, \quad \rho_{9}=\frac{[k l-(k-l+1)]}{(d+l-5)} \\
& \rho_{10}=\frac{k l}{(d+k-4)}, \quad \rho_{11}=\frac{[(d+k-4)-k l]}{(d+k-4)(d+l-5)}
\end{aligned}
$$



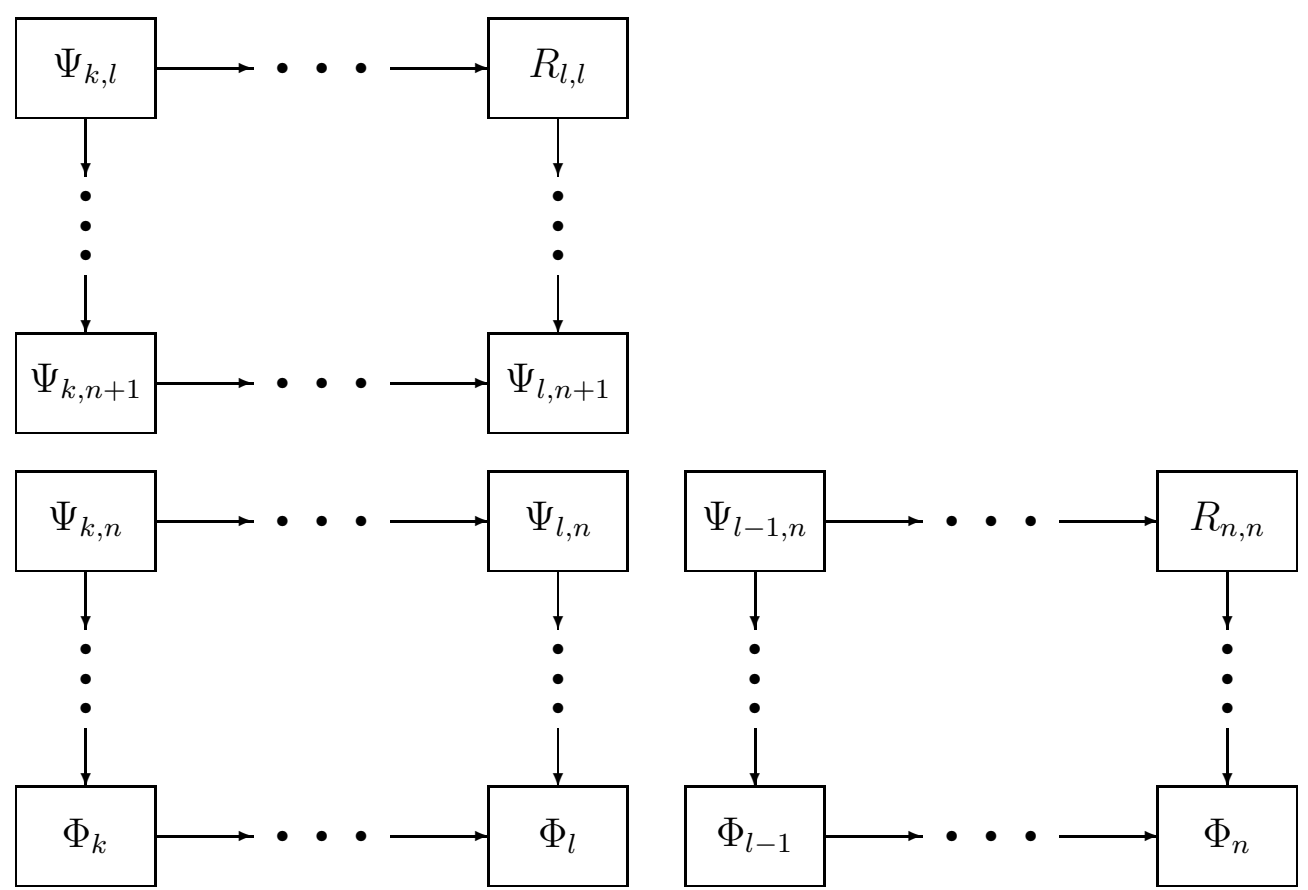

Figure 11: Example of non-unitary partially massless limit in $d S$ space

$$
\begin{aligned}
& \delta_{1} \Psi_{\mu \nu}{ }^{\left(a_{k}\right),\left(b_{n}\right)}=-\frac{d_{k, n}}{(k-n+2)(d+n-5)} \\
& {\left[(k-n+1) \xi_{[\mu}{ }^{\left(a_{k}\right),\left(b_{n-1}\right.} e_{\nu]}^{\left.b_{1}\right)}-e_{[\nu}^{\left(a_{1}\right.} \xi_{\mu]}{ }^{\left.a_{k-1}\right)\left(b_{1}, b_{n-1}\right)}+\right.} \\
& +\rho_{1} g^{\left(a_{1} a_{2}\right.} \xi_{[\mu, \nu]}^{\left.a_{k-2}\right)\left(b_{1}, b_{n-1}\right)}+\rho_{4} g^{\left(a_{1} a_{2}\right.} \xi_{[\mu}^{\left.a_{k-2}\right)\left(b_{1} b_{2}, b_{n-2}\right)}{ }_{\nu]}+ \\
& \left.+\rho_{2} g^{\left(a _ { 1 } \left(b_{1}\right.\right.} \xi_{[\mu, \nu]}^{\left.\left.a_{k-1}\right), b_{n l-1}\right)}+\rho_{3} g^{\left(a _ { 1 } \left(b_{1}\right.\right.} \xi_{[\mu}^{\left.\left.a_{k-1}\right) b_{2}, b_{n-2}\right)} \nu\right]+ \\
& \left.+\rho_{5} g^{\left(b_{1} b_{2}\right.} \xi_{[\mu}^{\left.\left(a_{k}\right), b_{n-2}\right)} \nu\right] \\
& \rho_{1}=\frac{2}{(d+k+n-4)}, \quad \rho_{2}=-\frac{(k-n)}{(d+k+n-4)}, \quad \rho_{3}=\frac{(d+2 k-4)}{(d+2 n-6)(d+k+n-4)} \\
& \rho_{4}=-\frac{4}{(d+2 n-6)(d+k+n-4)}, \quad \rho_{5}=-\frac{2(k-n+1)}{(d+2 n-6)} \\
& \delta_{1} \Omega_{\mu \nu}{ }^{\left(a_{k}\right),\left(b_{n}\right), c}=\frac{d_{k, n}}{(k-n+2)(d+n-4)} \\
& {\left[(k-n+1) e_{[\mu}{ }^{\left(b_{1}\right.} \eta_{\nu]}{ }^{\left.\left(a_{k}\right), b_{n-1}\right), c}-e_{[\mu}{ }^{\left(a_{1}\right.} \eta_{\nu]}{ }^{\left.a_{k-1}\right)\left(b_{1}, b_{n-1}\right), c}+\right.} \\
& +\rho_{1} g^{\left(a_{1} a_{2}\right.} \eta_{[\mu, \nu]}^{\left.a_{k-2}\right)\left(b_{1}, b_{n-1}\right), c}+\rho_{2} g^{\left(a_{1} a_{2}\right.} \eta_{[\mu}^{{ }^{\left.a_{k-2}\right)\left(b_{1} b_{2}, b_{n-2}\right)}{ }_{\nu]}^{c}+}+ \\
& +\rho_{3} g^{\left(a_{1} a_{2}\right.} \eta_{[\mu}^{\left.a_{k-2}\right) c\left(b_{1}, b_{n-1}\right)}{ }_{\nu]}+\rho_{4} g^{\left(a_{1} a_{2}\right.} \eta_{[\mu}^{\left.a_{k-2}\right)\left(b_{1} b_{2}, b_{n-2}\right) c}{ }_{\nu]}+ \\
& +\rho_{5} g^{\left(a _ { 1 } \left(b_{1}\right.\right.} \eta_{[\mu, \nu]}^{\left.\left.a_{k-1}\right), b_{n-1}\right), c}+\rho_{6} g^{\left(a _ { 1 } \left(b_{1}\right.\right.} \eta_{[\mu}{ }^{\left.\left.a_{k-1}\right) b_{2}, b_{n-2}\right)}{ }_{\nu]}^{c}+ \\
& +\rho_{7} g^{\left(a _ { 1 } \left(b_{1}\right.\right.} \eta_{[\mu}^{\left.\left.a_{k-1}\right) b_{2}, b_{n-2}\right) c}{ }_{\nu]}+\rho_{8} g^{\left(a _ { 1 } \left(b_{1}\right.\right.} \eta_{[\mu}^{\left.\left.a_{k-1}\right) c, b_{n-1}\right)}{ }_{\nu]}+
\end{aligned}
$$




$$
\begin{aligned}
& +\rho_{9} g^{c\left(a_{1}\right.} \eta_{[\mu}^{\left.a_{k-1}\right)\left(b_{1}, b_{n-1}\right)}{ }_{\nu]}+\rho_{10} g^{c\left(b_{1}\right.} \eta_{[\mu}^{\left.\left(a_{k}\right), b_{n-1}\right)}{ }_{\nu]}+ \\
& \left.+\rho_{11} g^{\left(b_{1} b_{2}\right.} \eta_{[\mu}^{\left.\left(a_{k}\right), b_{n-2}\right)}{ }_{\nu]}^{c}+\rho_{12} g^{\left(b_{1} b_{2}\right.} \eta_{[\mu}{ }^{\left.\left(a_{k}\right), b_{n-2}\right) c}{ }_{\nu]}\right] \\
& \rho_{1}=-\frac{2}{(d+k+n-4)}, \quad \rho_{5}=\frac{(k-n)}{(d+k+n-4)}, \quad \rho_{9}=-\frac{1}{(d+n-5)} \\
& \rho_{2}=\frac{4}{(d+2 n-6)(d+k+n-4)}, \quad \rho_{3}=\frac{2}{(d+n-5)(d+k+n-4)} \\
& \rho_{4}=-\frac{4}{(d+n-5)(d+2 n-6)(d+k+n-4)}, \quad \rho_{6}=-\frac{(d+2 k-4)}{(d+2 n-6)(d+k+n-4)} \\
& \rho_{7}=\frac{(d+2 k-4)}{(d+n-5)(d+2 n-6)(d+k+n-4)}, \quad \rho_{8}=-\frac{(k-n)}{(d+n-5)(d+k+n-4)}, \\
& \rho_{10}=\frac{(k-n+1)}{(d+n-5)}, \quad \rho_{11}=\frac{2(k-n+1)}{(d+2 n-6)}, \quad \rho_{12}=-\frac{2(k+n-1)}{(d+n-5)(d+2 n-6)} \\
& Y(m+1, n+1) \Leftrightarrow Y(m, n+1) \text {. Complete form for Formula (27) in Section } 3 \text {. } \\
& \delta_{1} \Psi_{\alpha \beta}\left(a_{m}\right),\left(b_{n}\right)=-\frac{c_{m, n}}{(d+m-4)}\left[e_{[\alpha}^{\left(a_{1}\right.} \xi_{\beta]}^{\left.a_{m-1}\right),\left(b_{n}\right)}+\rho_{1} g^{\left(a_{1} a_{2}\right.} \xi_{[\alpha, \beta]}^{\left.a_{m-2}\right),\left(b_{n}\right)}+\right. \\
& \left.\left.+\rho_{2} g^{\left(a_{1} a_{2}\right.} \xi_{[\alpha}^{\left.a_{m-2}\right)\left(b_{1}, b_{n-1}\right)} \beta\right]+\rho_{3} g^{\left(a _ { 1 } \left(b_{1}\right.\right.} \xi_{[\alpha}^{\left.\left.a_{m-1}\right), b_{n-1}\right)} \beta\right] \\
& \rho_{1}=\frac{2}{(d+2 m-4)}, \quad \rho_{2}=-\frac{2}{(d+2 m-4)(d+m+n-4)}, \quad \rho_{3}=\frac{1}{(d+m+n-4)} \\
& \delta_{1} \Omega_{\mu \nu}{ }^{\left(a_{m}\right),\left(b_{n}\right), c}=-\frac{c_{m, n}}{(d+m-3)}\left[e_{[\mu}{ }^{\left(a_{1}\right.} \eta_{\nu]}{ }^{\left.a_{m-1}\right),\left(b_{n}\right), c}+\rho_{1} g^{\left(a_{1} a_{2}\right.} \eta_{\mu, \nu}{ }^{\left.a_{m-2}\right),\left(b_{n}\right), c}+\right. \\
& +\rho_{2} g^{\left(a_{1} a_{2}\right.} \eta_{[\mu}^{\left.a_{m-2}\right)\left(b_{1}, b_{n-1}\right)}{ }_{\nu]}^{c}+\rho_{3} g^{\left(a_{1} a_{2}\right.} \eta_{[\mu}^{\left.a_{m-2}\right)\left(b_{1}, b_{n-1}\right) c}{ }_{\nu]}+ \\
& +\rho_{7} g^{\left(a_{1} a_{2}\right.} \eta_{[\mu}^{\left.a_{m-2}\right) c,\left(b_{n}\right)}{ }_{\nu]}+\rho_{4} g^{\left(a _ { 1 } \left(b_{1}\right.\right.} \eta_{[\mu}^{\left.\left.a_{m-1}\right), b_{n-1}\right)}{ }_{\nu]}^{c}+ \\
& \left.+\rho_{5} g^{\left(a _ { 1 } \left(b_{1}\right.\right.} \eta_{[\mu}^{\left.\left.a_{m-1}\right), b_{n-1}\right) c}{ }_{\nu]}+\rho_{6} g^{c\left(a_{1}\right.} \eta_{\mu}{ }^{\left.a_{m-1}\right),\left(b_{n}\right)}{ }_{\nu]}\right] \\
& \rho_{1}=\frac{2}{(d+2 m-4)}, \quad \rho_{2}=-\frac{2}{(d+2 m-4)(d+m+n-4)}, \quad \rho_{4}=\frac{1}{(d+m+n-4)} \\
& \rho_{3}=\frac{2}{(d+m-4)(d+2 m-4)(d+m+n-4)}, \quad \rho_{7}=-\frac{2}{(d+m-4)(d+2 m-4)} \\
& \rho_{5}=-\frac{1}{(d+m-4)(d+m+n-4)}, \quad \rho_{6}=\frac{1}{(d+m-4)}
\end{aligned}
$$




\section{References}

[1] Yu. M. Zinoviev "Towards frame-like gauge invariant formulation for massive mixed symmetry bosonic fields", Nucl. Phys. B812 (2009) 46, arXiv:0809.3287.

[2] M. A. Vasiliev "'Gauge' form of description of massless fields with arbitrary spin", Sov. J. Nucl. Phys. 32 (1980) 439.

[3] V. E. Lopatin, M. A. Vasiliev "Free massless bosonic fields of arbitrary spin in $d$ dimensional de sitter space", Mod. Phys. Lett. A3 (1988) 257.

[4] M. A. Vasiliev "Free massless fermionic fields of arbitrary spin in d-dimensional de sitter space", Nucl. Phys, B301 (1988) 26.

[5] K. B. Alkalaev, O. V. Shaynkman, M. A. Vasiliev "On the Frame-Like Formulation of Mixed-Symmetry Massless Fields in (A)dS(d)", Nucl. Phys. B692 (2004) 363, arXiv:hep-th/0311164.

[6] K. B. Alkalaev "Two-column higher spin massless fields in AdS(d)", Theor. Math. Phys. 140 (2004) 1253, arXiv:hep-th/0311212.

[7] K. B. Alkalaev, O. V. Shaynkman, M. A. Vasiliev "Lagrangian Formulation for Free Mixed-Symmetry Bosonic Gauge Fields in (A)dS(d)", JHEP 0508 (2005) 069, arXiv:hep-th/0501108.

[8] K. B. Alkalaev, O. V. Shaynkman, M. A. Vasiliev "Frame-like formulation for free mixed-symmetry bosonic massless higher-spin fields in AdS(d)", arXiv:hep-th/0601225.

[9] E. D. Skvortsov "Frame-like Actions for Massless Mixed-Symmetry Fields in Minkowski space", Nucl. Phys. B808 (2009) 569, arXiv:0807.0903.

[10] L. Brink, R. R. Metsaev, M. A. Vasiliev "How massless are massless fields in AdS $S_{d}$, Nucl. Phys. B586 (2000) 183, arXiv:hep-th/0005136.

[11] I. L. Buchbinder, V. A. Krykhtin "Gauge invariant Lagrangian construction for massive bosonic higher spin fields in D dimensions", Nucl. Phys. B727 (2005) 537, arXiv:hep-th/0505092.

[12] I. L. Buchbinder, V. A. Krykhtin, L .L. Ryskina, H. Takata "Gauge invariant Lagrangian construction for massive higher spin fermionic fields", Phys. Lett. B641 (2006) 386, arXiv:hep-th/0603212.

[13] I. L. Buchbinder, V. A. Krykhtin, P. M. Lavrov "Gauge invariant Lagrangian formulation of higher spin massive bosonic field theory in AdS space", Nucl. Phys. B762 (2007) 344, arXiv:hep-th/0608005.

[14] I. L. Buchbinder, V. A. Krykhtin, A. A. Reshetnyak "BRST approach to Lagrangian construction for fermionic higher spin fields in (A)dS space", Nucl. Phys. B787 (2007) 211, arXiv:hep-th/0703049. 
[15] I. L. Buchbinder, V. A. Krykhtin, H. Takata "Gauge invariant Lagrangian construction for massive bosonic mixed symmetry higher spin fields", Phys. Lett. B656 (2007) 253, arXiv:0707.2181.

[16] P. Yu. Moshin, A. A. Reshetnyak "BRST approach to Lagrangian formulation for mixedsymmetry fermionic higher-spin fields", JHEP 10 (2007) 040, arXiv:0707.0386.

[17] I. L. Buchbinder, V. A. Krykhtin, L. L. Ryskina "BRST approach to Lagrangian formulation of bosonic totally antisymmetric tensor fields in curved space", Mod. Phys. Lett. A24 (2009) 401, arXiv:0810.3467.

[18] I. L. Buchbinder, V. A. Krykhtin, L. L. Ryskina "Lagrangian formulation of massive fermionic totally antisymmetric tensor field theory in $A d S_{d}$ space", arXiv:0902.1471.

[19] S. M. Klishevich, Yu. M. Zinoviev "On electromagnetic interaction of massive spin-2 particle", Phys. Atom. Nucl. 61 (1998) 1527, arXiv:hep-th/9708150.

[20] Yu. M. Zinoviev "On Massive High Spin Particles in (A)dS", arXiv:hep-th/0108192.

[21] P. de Medeiros "Massive gauge-invariant field theories on spaces of constant curvature", Class. Quant. Grav. 21 (2004) 2571, arXiv:hep-th/0311254.

[22] R. R. Metsaev "Gauge invariant formulation of massive totally symmetric fermionic fields in (A)dS space", Phys. Lett. B643 (2006) 205-212, arXiv:hep-th/0609029.

[23] Yu. M. Zinoviev "Frame-like gauge invariant formulation for massive high spin particles", Nucl. Phys. B808 (2009) 185, arXiv:0808.1778.

[24] I. L. Buchbinder, A. V. Galajinsky "Quartet unconstrained formulation for massive higher spin fields", JHEP 0811 (2008) 081, arXiv:0810.2852.

[25] Yu. M. Zinoviev "Note on antisymmetric spin-tensors", JHEP 04 (2009) 035, arXiv:0903.0262.

[26] Yu. M. Zinoviev "Frame-like gauge invariant formulation for mixed symmetry fermionic fields", Nucl. Phys. B (2009), arXiv:0904.0549.

[27] S. Deser, A. Waldron "Gauge Invariance and Phases of Massive Higher Spins in (A)dS", Phys. Rev. Lett. 87 (2001) 031601, arXiv:hep-th/0102166.

[28] S. Deser, A. Waldron "Partial Masslessness of Higher Spins in (A)dS", Nucl. Phys. B607 (2001) 577, arXiv:hep-th/0103198.

[29] S. Deser, A. Waldron "Null Propagation of Partially Massless Higher Spins in (A) $d S$ and Cosmological Constant Speculations", Phys. Lett. B513 (2001) 137, arXiv:hep-th/0105181.

[30] E. D. Skvortsov, M. A. Vasiliev "Geometric Formulation for Partially Massless Fields", Nucl. Phys. B756 (2006) 117, arXiv:hep-th/0601095. 